\title{
Pattern formation in a reaction-diffusion rumor propagation system with Allee effect and time delay
}

\author{
Linhe Zhu ( $\nabla$ zlhnuaa@126.com ) \\ Jiangsu University https://orcid.org/0000-0002-2457-4821 \\ Le He \\ Jiangsu University
}

\section{Research Article}

Keywords: Information propagation, Turing pattern, Reaction-diffusion system, Periodic diffusion, Time delay

Posted Date: April 30th, 2021

DOI: https://doi.org/10.21203/rs.3.rs-239109/v1

License: (1) This work is licensed under a Creative Commons Attribution 4.0 International License. Read Full License

Version of Record: A version of this preprint was published at Nonlinear Dynamics on January 13th, 2022. See the published version at https://doi.org/10.1007/s11071-021-07106-7. 


\title{
Pattern formation in a reaction-diffusion rumor propagation system with Allee effect and time delay
}

Linhe Zhu ${ }^{1}$, a) and Le He ${ }^{1}$

School of Mathematical Sciences, Jiangsu University, Zhenjiang, 212013, China.

(Dated: 21 January 2021)

\begin{abstract}
This paper analyzes the diffusion behavior of the suspicious and the infected cabins in cyberspace by establishing a rumor propagation reaction diffusion model with Allee effect and time delay. The Turing instability conditions of the system under various conditions are emphatically studied. After considering the delay effect of rumor propagation systems, we have studied the correlation between the stability of the system under the influence of small time delay and the homogeneous system near the equilibrium point, and the critical condition of the delay-induced spatial instability is given. Further considering the possibility of diffusion coefficient changing with time, the critical parameter curves of stability and instability of approximate systems are given by means of Floquet theory, and the necessary conditions of Turing-instability of periodic coefficient are studied. In the numerical simulations, we find that the variation of diffusion coefficient will change the pattern type, and the periodical diffusion behavior will affect the arrangement of the crowd gathering area in the pattern.
\end{abstract}

Keywords: Information propagation, Turing pattern, Reaction-diffusion system, Periodic diffusion, Time delay.

\section{INTRODUCTION}

Nowadays, social network environment is characterized by fast information dissemination speed and low access cost, making it a stepping stone for false information and influencing public opinion ${ }^{1}$. Rumor is the main form of false information, which will cause great misdirection and harm to the public opinion environment. Therefore, it is a very important topic to study the spread of information among people and timely control the spread of rumors.

Research on information or rumor propagation started a long time ago. Daley put forward the random rumor model in $1965^{2}$, and Munaki established the MK model in $1973^{3}$. Due to the high similarity between rumor propagation and infectious disease propagation, many scholars have tried to improve the traditional infectious disease models and established the cabin ordinary differential equation models of rumor propagation ${ }^{4-6}$. In Huo's work ${ }^{4}$, the population was divided into three categories: the ignorant population $X(t)$, the aware population $X_{m}(t)$ and spreaders $Y(t)$, as the accumulative consciousness mechanism $M(t)$ was further introduced. However, in recent years, with the in-depth development of complex network research and the high superiority of network structure in describing user structure in social networks, the establishment of rumor propagation dynamic model based on different network topologies began to rise ${ }^{7-12,39,40}$. According to Huo's work, rumor propagation dynamics in homogeneous networks wass studied, and the global stability of the internal equilibrium of the model was proved by using Lasalle's invariant principle ${ }^{12}$. In the work of Wang, the process of information transmission in multi-layer social networks was studied, and the results showed that the success rate of rumor spreading was positively correlated with the node degree and the number of layers of initial nodes ${ }^{7}$. Based on the two-layer network, Xiao used the Markov chain method to study the coupling propagation process of multiple information, and the correlation coefficient between the networks would negatively affect the information diffusion threshold ${ }^{8}$. In the process of rumor propagation, the differences between different individuals or nodes are constantly concerned. For example, heterogeneous networks represent huge differences in node degree distribution, and rumor propagation based on this has been studied in some documents ${ }^{11}$. In the work of Yang, a connection was established between the propagation probability of rumors and node degrees, so as to take individual differences into consideration ${ }^{10}$. Besides ordinary differential equation models, the stochastic differential equation method after introducing randomness can also describe the rumor propagation process well ${ }^{13}$. Due to the high correlation between epidemic transmission and related information in practice and the similarity of transmission process, some work have tried to establish the coupling model of information transmission and disease transmission. ${ }^{14-16}$. It reveals the mechanism of the interaction between disease and related consciousness. These works also reflect the important potential practical value of the research on the dynamics of information and rumor propagation.

Most of the above work is to establish the ordinary differential equation or difference equation model of rumor spread under different backgrounds and express its spatial relationship by using the static network structure. These works cannot well reflect

a)Electronic mail: zlhnuaa@126.com 
the mobility of users in cyberspace and the stability of rumor propagation in space. Therefore, we would like to make more attempts to use partial differential equations to build rumor modeling and focus on studying Turing instability conditions. In 1952, Turing described a phenomenon of spatial instability ${ }^{17}$, which became known as the Turing pattern later. Study of Turing pattern has been widely used in molecular chemistry and physics in the early days ${ }^{19-22,41}$. However, with the extensive development of biomathematics, in the predator-prey model ${ }^{23,25,28}$ and parasite-host model ${ }^{24}$, it is employed to delineate the spatial distribution instablity of the population. In the subsequent population model, the reaction-diffusion process with cross diffusion is the main cause of the pattern phenomenon ${ }^{23-25}$. After introducing the time delay in some models, it will also become one of the reasons for the appearance of pattern ${ }^{26,27}$. Some scholars have studied the phenomenon of speckle pattern on the network structure and obtained the corresponding conditions ${ }^{34,35}$. Unlike many previous assumptions of the constant diffusion coefficients, in the work of Djouda ${ }^{29}$, the population reactivity diffusion model of mycelium and insects was established, and its diffusion coefficient had periodic characteristics. By establishing the linear approximate equation of perturbation near the equilibrium point, they try to derive the condition of pattern driven by period. The rumor propagation model established in this paper will follow this idea and improve some of the results.

The structure after this paper is as follows: In the second section, we establish a spatiotemporal model with time-delay and periodic propagation of rumor. In the third section, we will analyze the local stability of the equilibrium point in the time dimension in the case of non-diffusion. In the fourth section, the conditions of Turing instability in the spatiotemporal model are given. In the fifth section, extensive numerical simulations are carried out. Finally, we put forward the conclusion of our work in the sixth section.

\section{MODELLING}

Based on previous work, this section attempts to establish a series of suspicious-infected-recovered spatiotemporal dynamics models describing rumor propagation in information cyberspace, and focuses on its spatial stability and Turing branch phenomenon. $S$ (Suspicious) refers to people who have not been informed of rumors or doubt the authenticity of rumors, $I$ (Infected) are rumor-believers, that is, people who have "infected" by rumors and begun to spread, $R$ (Recovered) refers to people who no longer believe in rumors or lose interest in them. The average field model of rumor propagation considering only the time dimension is shown as:

$$
\left\{\begin{array}{l}
\frac{d S(t)}{d t}=r S(t)\left(1-\frac{S(t)}{K}\right)\left(\frac{S(t)}{A}-1\right)-\beta S(t) I(t), \\
\frac{d I(t)}{d t}=\beta S(t) I(t)-\mu I^{2}(t), \\
\frac{d R(t)}{d t}=\mu I^{2}(t),
\end{array}\right.
$$

where $S(0)>0, I(0)>0$. The first term of the first equation of model (1) represents the process of new users entering the information network space, which makes the number of users grow naturally. $r S\left(1-\frac{S}{K}\right)$ is a Logistic growth model, where $r$ refers to the intrinsic growth rate, $K$ is the maximum user capacity of information cyberspace. $\left(\frac{S}{A}-1\right)$ represents the Allee effect of growth, where $A$ refers to the critical spatial carrying capacity ${ }^{36-38}$. The Allee effect truly reflects that when the number of users in a certain information cyberspace is low, the number of new users attracted is also relatively small. $\beta$ defines the rate of rumor spread, that is, the probability that a rumor believer communicates with a suspicious one and makes it a rumor believer in a unit time. $\mu$ refers to the rate of refuting rumors, which means that when there are two rumour believers communicating, they have the probability to check the authenticity of the rumors so that they no longer believe in rumors or lose interest in spreading.

In order to simplify the study of model (1), we make a simple linear transformation and redefine the parameters: $\beta_{\text {new }}=$ $\frac{A K}{r} \beta, \quad \mu_{\text {new }}=\frac{A K}{r} \mu, \quad t_{\text {new }}=\frac{r}{A K} t$. Meanwhile, since the dynamic behavior of various groups in the model can be completely determined by the behavior of $S$ and $I$, model (1) can be simplified to the following forms:

$$
\left\{\begin{array}{l}
\frac{d S(t)}{d t}=S(t)(K-S(t))(S(t)-A)-\beta S(t) I(t), \\
\frac{d I(t)}{d t}=\beta S(t) I(t)-\mu I^{2}(t) .
\end{array}\right.
$$

Here, for ease of writing, we have omitted the subscript "new" of the new parameters. In the following passage, when we add a new term to the model, the coefficient before the term is naturally changed to $\frac{A K}{r}$ times the original.

We extend the model to spatial latitude within a fixed bounded domain $\Omega \subset \mathbb{R}^{2}$. Considering the cross-diffusion behavior of 
users in cyberspace, the model is as follows:

$$
\left\{\begin{array}{l}
\frac{\partial S(t, x)}{\partial t}=D_{11}(t) \Delta S(t, x)+D_{12}(t) \Delta I(t, x)+S(t, x)(K-S(t, x))(S(t, x)-A)-\beta S(t, x) I(t, x), t>0, x \in \Omega \\
\frac{\partial I(t, x)}{\partial t}=D_{21}(t) \Delta S(t, x)+D_{22}(t) \Delta I(t, x)+\beta S(t, x) I(t, x)-\mu I^{2}(t, x), t>0, x \in \Omega \\
\left.\frac{\partial S(t, x)}{\partial \vec{n}}\right|_{\partial \Omega}=\left.\frac{\partial I(t, x)}{\partial \vec{n}}\right|_{\partial \Omega}=0, t \geq 0, x \in \partial \Omega \\
S(0, x)>0, \quad I(0, x)>0, \quad x \in \bar{\Omega} .
\end{array}\right.
$$

$D_{11}(t)>0, D_{22}(t)>0$ represent the diffusion coefficient, describing the dynamic behavior of all groups of people in cyberspace based on their own growth. $D_{12}(t)$ and $D_{21}(t)$ represent the cross diffusion coefficient and describe the spatial trends caused by the existence of other populations. $\Delta$ is Laplace operator. It is worth noting that when $D_{11}(t) D_{22}(t)-D_{12}(t) D_{12}(t)>0$, the flow of people in the spatial domain depends more on their own density, and in subsequent studies, we will base on this assumption. $n$ is the outward unit normal vector on boundary $\Omega$. Our study will adopt zero flux Newman boundary conditions and positive initial conditions. This condition indicates that there is no external input or internal outflow at the system boundary.

In addition, in the background of rumor propagation, time delay is always considered to be an important factor in describing the system more accurately. In the study of the stability of the reaction diffusion system, the delay is also an important reason for the phenomenon of Turing pattern ${ }^{26,27}$. On the basis of model (3), we consider two improved time-delay as follows.

$$
\left\{\begin{array}{l}
\frac{\partial S(t, x)}{\partial t}=D_{11}(t) \Delta S(t, x)+D_{12}(t) \Delta I(t, x)+S(t, x)(K-S(t, x))(S(t, x)-A)-\beta S\left(t-\tau_{2}\right) I\left(t-\tau_{2}, x\right), t>0, x \in \Omega \\
\frac{\partial I(t, x)}{\partial t}=D_{21}(t) \Delta S(t, x)+D_{22}(t) \Delta I(t, x)+\beta S\left(t-\tau_{2}, x\right) I\left(t-\tau_{2}, x\right)-\mu I^{2}\left(t-\tau_{1}, x\right), t>0, x \in \Omega
\end{array}\right.
$$

with homogeneous Neumann boundary conditions

$$
\frac{\partial S}{\partial \vec{n}}(t, x)=\frac{\partial I}{\partial \vec{n}}(t, x)=0, t \geq 0, x \in \partial \Omega,
$$

and initial conditions

$$
\left\{\begin{array}{l}
S(t, x)=S_{0}(t, x) \geq 0 \text { and } \not \equiv 0,(t, x) \in\left[-\tau_{0}, 0\right] \times \bar{\Omega} \\
I(t, x)=I_{0}(t, x) \geq 0 \text { and } \not \equiv 0,(t, x) \in\left[-\tau_{0}, 0\right] \times \bar{\Omega}
\end{array}\right.
$$

where $\tau_{0}=\max \left\{\tau_{1}, \tau_{2}\right\}$ and $\tau_{1}, \tau_{2}$ both represent a small positive time delay in the above model. Firstly, $\tau_{1}$ explains a time lag between the rumor reader checking the information and the refutation; Secondly, $\tau_{2}$ reflects that the suspicious who accept and believe the rumor has a certain delay until they begin to spread.

\section{LOCAL STABILITY ANALYSIS OF NON-DIFFUSION CONDITIONS}

One of the necessary conditions for Turing bifurcation is that the corresponding ordinary differential equation system is locally stable at the equilibrium point. In this section, the stability conditions of the positive equilibrium point of model (2) will be analyzed, which provides the necessary conditions for Turing instability of the reaction-diffusion rumor propagation system.

Model (2) has five equilibrium points. Among them, $\mathrm{P}_{1}=(K, 0), \mathrm{P}_{2}=(A, 0), \mathrm{P}_{3}=(0,0)$ are the rumor-free equilibrium points. The rumor-spreading equilibrium points are

$$
\begin{aligned}
& \mathrm{P}_{4}=\left(\frac{1}{2}\left(p-\sqrt{p^{2}-4 q}\right), \frac{\beta}{2 \mu}\left(p-\sqrt{p^{2}-4 q}\right)\right), \\
& \mathrm{P}_{5}=\left(\frac{1}{2}\left(p+\sqrt{p^{2}-4 q}\right), \frac{\beta}{2 \mu}\left(p+\sqrt{p^{2}-4 q}\right)\right),
\end{aligned}
$$

under the conditions that $p>0, p^{2}-4 q>0$, where $p=K+A-\frac{\beta^{2}}{\mu}, q=A K$. The Jacobian matrix at the equilibrium point $\mathrm{P}=\left(S^{*}, I^{*}\right)$ of model (2) is defined as $J(\mathrm{P})$ :

$$
J(\mathrm{P})=\left(\begin{array}{cc}
S^{*}\left(2 \frac{\beta^{2}}{\mu}-K-A\right)+2 A K & -\beta S^{*} \\
S^{*} \frac{\beta^{2}}{\mu} & -\beta S^{*}
\end{array}\right) .
$$


The characteristic equation of model (2) can be obtained:

$$
\lambda^{2}-\operatorname{tr}(J) \lambda+\operatorname{det}(J)=0,
$$

where $\operatorname{tr}(J)=S^{*}\left(2 \frac{\beta^{2}}{\mu}-A-K-\beta\right)+2 A K, \operatorname{det}(J)=-\beta S^{*}\left(S^{*}\left(\frac{\beta^{2}}{\mu}-A-K\right)+2 A K\right)$. When the eigenvalues of the characteristic equation are satisfied with negative real parts, the system is locally asymptotically stable near the point P. Because of $\lambda_{1} \lambda_{2}=$ $\operatorname{det}(J)$ and $\lambda_{1}+\lambda_{2}=\operatorname{tr}(J)$, the judgment of the local stability of the system can be converted to discuss the positive and negative properties of determinant and trace. Further, we will analyze the sufficient conditions for the local stable state of the rumorspreading equilibrium points based on the size relationship between $\beta$ and $\mu$.

- $\beta \geq \mu$ : To make $\operatorname{det}(J)>0$ in this case, just satisfy the $\operatorname{tr}(J)<0$. Therefore, the conditions that $P_{4}$ and $P_{5}$ are stable are $\operatorname{tr}\left(J\left(\mathrm{P}_{4}\right)\right)<0$ and $\operatorname{tr}\left(J\left(\mathrm{P}_{5}\right)\right)<0$. Satisfied with the above constraints, the extra constraints for both two rumor- spreading equilibrium points are stable as follows:

$$
\left\{\begin{array}{l}
\frac{2 \beta^{2}}{\mu}<(K+A+\beta) \\
\frac{2 \beta^{2}}{\mu}>\frac{(K-A-\beta)(K-A+\beta)}{(K+A-\beta)} \\
4 q<p^{2}+\left(1-\frac{\beta}{\mu}\right) \beta p .
\end{array}\right.
$$

Let $\operatorname{tr}\left(J\left(\mathrm{P}_{4}\right)\right)>0$ and $\operatorname{tr}\left(J\left(\mathrm{P}_{5}\right)\right)<0$, the conditions that $\mathrm{P}_{4}$ is instability and $\mathrm{P}_{5}$ is stability are

$$
\frac{2 \beta^{2}}{\mu}<\min \left\{(K+A+\beta), \frac{(K-A-\beta)(K-A+\beta)}{(K+A-\beta)}\right\} .
$$

Further, we consider the conditions that $\mathrm{P}_{5}$ is instable and $\mathrm{P}_{4}$ is stable. But such conditions are contradictory and therefore this case does not exist.

- $\beta<\mu$ : To make $\operatorname{tr}(\mathrm{J})>0$ in this case, just satisfy the $\operatorname{det}(\mathrm{J})<0$.

$$
\begin{array}{ll}
\text { For } \quad \mathrm{P}_{4}: & S_{4}^{*}\left(\frac{\beta^{2}}{\mu}-K-A\right)+2 A K=\frac{1}{2} \sqrt{p^{2}-4 q}\left(p-\sqrt{p^{2}-4 q}\right)>0 \Rightarrow \operatorname{det}\left(J\left(\mathrm{P}_{4}\right)\right)<0 . \\
\text { For } \quad \mathrm{P}_{5}: & S_{5}^{*}\left(\frac{\beta^{2}}{\mu}-K-A\right)+2 A K=\frac{1}{2} \sqrt{p^{2}-4 q}\left(-p-\sqrt{p^{2}-4 q}\right)<0 \Rightarrow \operatorname{det}\left(J\left(\mathrm{P}_{5}\right)\right)>0 .
\end{array}
$$

Therefore, in this case, as long as the rumor-spreading equilibrium points exist, there is a conclusion that the $\mathrm{P}_{4}$ is unstable and $\mathrm{P}_{5}$ is local asymptotic stability.

To simplify the later study, we will analyze the Turing bifurcation of rumor propagation models when $\beta<\mu$ holds. Since only $\mathrm{P}_{5}$ are locally asymptotically stable, the Turing pattern will only appear near this equilibrium point. We write the single stable point $\mathrm{P}_{5}$ as $\mathrm{P}=\left(S^{*}, I^{*}\right)$ to simplify the expression of following formulas.

\section{TURING INSTABILITY ANALYSIS}

\section{A. System of constant diffusion coefficient}

Now, we consider diffusion coefficients as constants, that is, diffusion coefficients are time-independent: $D_{11}(t)=d_{11}$, $D_{12}(t)=d_{12}, D_{21}(t)=d_{21}$ and $D_{22}(t)=d_{22}$. Model (3) of constant diffusion coefficients is simplified as follows:

$$
\left\{\begin{array}{l}
\frac{\partial S}{\partial t}=d_{11} \Delta S+d_{12} \Delta I+S(K-S)(S-A)-\beta S I \\
\frac{\partial I}{\partial t}=d_{21} \Delta S+d_{22} \Delta I+\beta S I-\mu I^{2} .
\end{array}\right.
$$

Based on the assumption that $\beta<\mu$, the rumor- spreading equilibrium point $\mathrm{P}$ is local stable in the time dimension, and the necessary condition for the occurrence of Turing bifurcation is the instability of $\mathrm{P}$ in the presence of diffusion in space. The perturbation $\left(\delta_{1}, \delta_{2}\right)$ near the equilibrium point is expanded in the Fourier space:

$$
\begin{aligned}
& \delta_{1}(\tilde{\mathrm{x}}, \mathrm{t})=\sum_{k} c_{k}^{1} e^{\lambda_{k} t+i k \vec{x}} \\
& \delta_{2}(\tilde{\mathrm{x}}, \mathrm{t})=\sum_{k} c_{k}^{2} e^{\lambda_{k} t+i k \vec{x}}
\end{aligned}
$$


where $k$ and $\lambda$ stand for wave number and wave frequence respectively. Substituting the above equation into the linear approximation system of system (7), then the characteristic equation of system (7) is $|H(k)-\lambda I|=0$, where $H(k)=J-k^{2} D, D$ represents the diffusion coefficient matrix. The dispersion relation is obtained as

$$
\lambda_{k}^{2}-\operatorname{tr}(H(k)) \lambda_{k}+\Delta_{k}=0
$$

where $\operatorname{tr}(H(k))=\operatorname{tr}(J)-k^{2} \operatorname{tr}(D)<0, \Delta_{k}=\operatorname{det}(H(k))=\eta k^{4}+\theta k^{2}+\Delta_{0}$. Among them, $\eta=\operatorname{det}(D)>0, \theta=d_{21} j_{12}+d_{12} j_{21}-$ $d_{11} j_{22}-d_{22} j_{11}, j$ stands for the element of matrix $J(P)$. If for any $k$, the characteristic roots of system (7) all have a negative real part, then Turing pattern cannot be formed. Due to $\operatorname{tr}(H(k))<0$, the condition of the existence of eigenvalues with positive real parts of the system is transformed into: there exist some $k$, make $\Delta_{k}<0 . \Delta_{k}$ 's minimum point for $k$, namely the most dangerous modulus of the system $k_{c}$ is expressed as

$$
k_{c}^{2}=-\frac{\theta}{2 \eta} .
$$

According to $k_{c}$, the critical condition for the emergence of Turing bifurcation is as follows:

$$
\Delta_{k_{c}}=\Delta_{0}-\frac{\theta^{2}}{4 \eta}=0
$$

Ensure that $k^{2}$ nonnegative, $\Delta_{k_{c}}<0$, we can give the necessary conditions for the existence of diffusion-driven Turing instability: $\theta<0$ and $4 \eta \Delta_{0}<\theta^{2}$.

\section{B. System of time-delay}

In this section, we will analyze the Turing bifurcation conditions of system (4) under $\tau_{1}=0$ and $\tau_{2}=0$ respectively with the constant periodic coefficient. The equations are reduced to:

$$
\left\{\begin{array}{l}
\frac{\partial S}{\partial t}=d_{11} \Delta S+d_{12} \Delta I+S(K-S)(S-A)-\beta S I \\
\frac{\partial I}{\partial t}=d_{21} \Delta S+d_{22} \Delta I+\beta S I-\mu I^{2}\left(t-\tau_{1}\right)
\end{array}\right.
$$

and

$$
\left\{\begin{array}{l}
\frac{\partial S}{\partial t}=d_{11} \Delta S+d_{12} \Delta I+S(K-S)(S-A)-\beta S\left(t-\tau_{2}\right) I\left(t-\tau_{2}\right) \\
\frac{\partial I}{\partial t}=d_{21} \Delta S+d_{22} \Delta I+\beta S\left(t-\tau_{2}\right) I\left(t-\tau_{2}\right)-\mu I^{2}
\end{array}\right.
$$

where $\tau_{1}$ and $\tau_{2}$ represent the small time delays. In order to facilitate the analysis of the stability of the original system with time delay, the following theorem is given.

Theorem 1. Set up a constant coefficient reactive diffusion system in the following form:

$$
\left\{\begin{array}{l}
\frac{\partial X}{\partial t}=d_{11} \Delta X+d_{12} \Delta Y+F(X(t), Y(t))+\varphi(X(t), Y(t)), \\
\frac{\partial Y}{\partial t}=d_{21} \Delta X+d_{22} \Delta Y+G(X(t), Y(t))+\psi(X(t), Y(t)),
\end{array}\right.
$$

where $\varphi$ and $\psi$ have the first partial derivatives of $X$ and $Y$, and the system has an equilibrium point $P_{0}$. Replace the term without delay to the term with delay: $\varphi(X(t), Y(t)) \rightarrow \varphi(X(t-\tau), Y(t-\tau)), \psi(X(t), Y(t)) \rightarrow \psi(X(t-\tau), Y(t-\tau))$. When $\tau$ is very small, Taylor expansion is carried out for terms containing $\tau$, and the approximate system of linear terms $\left(X_{\text {new }}, Y_{\text {new }}\right)^{T}$ is taken to satisfy the following properties near $P_{0}$ :

(1) $\left[\begin{array}{c}\frac{\partial X_{n e w}}{\partial t} \\ \frac{\partial Y_{n e w}}{\partial t}\end{array}\right]=Q\left[\begin{array}{l}\frac{\partial X}{\partial t} \\ \frac{\partial Y}{\partial t}\end{array}\right]$

where $Q=\frac{1}{\left(1+\tau \frac{\partial \varphi}{\partial X}\right)\left(1+\tau \frac{\partial \psi}{\partial Y}\right)-\tau^{2} \frac{\partial \varphi}{\partial Y} \frac{\partial \psi}{\partial X}}\left[\begin{array}{cc}1+\tau \frac{\partial \psi}{\partial Y} & -\tau \frac{\partial \varphi}{\partial Y} \\ -\tau \frac{\partial \psi}{\partial X} & 1+\tau \frac{\partial \varphi}{\partial X}\end{array}\right]$. 
(2) Jacobian matrix at $P_{0}$ for systems with time delay: $J_{\text {new }}\left(P_{0}\right)=Q\left(P_{0}\right) J\left(P_{0}\right)$. Diffusion coefficient matrix at $P_{0}$ for delay systems: $D_{\text {new }}\left(P_{0}\right)=Q\left(P_{0}\right) D$. Let $H_{\text {new }}(k)=J_{\text {new }}\left(P_{0}\right)+k^{2} D_{\text {new }}\left(P_{0}\right)$ and it satisfies $H_{\text {new }}(k)=Q\left(P_{0}\right) H(k)$.

Proof. The system with time delay derived from the new system is as follows:

$$
\left\{\begin{array}{l}
\frac{\partial X}{\partial t}=d_{11} \Delta X+d_{12} \Delta Y+F(X(t), Y(t))+\varphi(X(t-\tau), Y(t-\tau)), \\
\frac{\partial Y}{\partial t}=d_{21} \Delta X+d_{22} \Delta Y+G(X(t), Y(t))+\psi(X(t-\tau), Y(t-\tau)) .
\end{array}\right.
$$

Taylor expansion is performed for $\varphi, \psi$ with $\tau$ as the variable, and the linear term is taken as

$$
\begin{aligned}
& \varphi(X(t-\tau), Y(t-\tau))=\varphi(X(t), Y(t))-\tau \frac{\partial \varphi(X(t), Y(t))}{\partial t}+o\left(\tau^{2}\right), \\
& \psi(X(t-\tau), Y(t-\tau))=\psi(X(t), Y(t))-\tau \frac{\partial \varphi(X(t), Y(t))}{\partial t}+o\left(\tau^{2}\right) .
\end{aligned}
$$

Write $M=\left(m_{i j}\right)_{2 \times 2}$ and define

$$
M \triangleq \tau \frac{\partial(\varphi, \psi)}{\partial(X, Y)}=\left[\begin{array}{ll}
\tau \frac{\partial \varphi}{\partial X} & \tau \frac{\partial \varphi}{\partial Y} \\
\tau \frac{\partial \psi}{\partial X} & \tau \frac{\partial \psi}{\partial Y}
\end{array}\right]
$$

In this case, we have $\tau \frac{\partial \varphi}{\partial t}=m_{11} \frac{\partial X}{\partial t}+m_{12} \frac{\partial Y}{\partial t}$ and $\tau \frac{\partial \psi}{\partial t}=m_{21} \frac{\partial X}{\partial t}+m_{22} \frac{\partial Y}{\partial t}$. The linear approximation term and the upper formula are substituted into the system, and the delay approximation system is arranged as follows:

$$
\left\{\begin{array}{c}
\left(1+m_{11}\right) \frac{\partial X}{\partial t}+m_{12} \frac{\partial Y}{\partial t}=F(X, Y)+\varphi(X, Y)+d_{11} \Delta X+d_{12} \Delta Y=\frac{\partial X_{\mathrm{old}}}{\partial t} \\
m_{21} \frac{\partial X}{\partial t}+\left(1+m_{22}\right) \frac{\partial Y}{\partial t}=G(X, Y)+\psi(X, Y)+d_{12} \Delta X+d_{22} \Delta Y=\frac{\partial Y_{\mathrm{old}}}{\partial t}
\end{array}\right.
$$

Obviously the following equation is true:

$$
\left[\begin{array}{c}
\frac{\partial X_{\text {old }}}{\partial t} \\
\frac{\partial Y_{\text {old }}}{\partial t}
\end{array}\right]=\left[\begin{array}{cc}
1+m_{11} & m_{12} \\
m_{21} & 1+m_{22}
\end{array}\right]\left[\begin{array}{c}
\frac{\partial X_{\text {new }}}{\partial t} \\
\frac{\partial Y_{\text {new }}}{\partial t}
\end{array}\right]
$$

Because $\varphi$ and $\psi$ have the first partial derivative of $X$ and $Y$ around $P_{0}$, we can assume that $\frac{\partial \varphi}{\partial X}, \frac{\partial \psi}{\partial Y}, \frac{\partial \varphi}{\partial Y}$ and $\frac{\partial \psi}{\partial X}$ are bounded in a neighborhood of $P_{0}$. So when $\tau$ is small enough, the determinant of the matrix in above equation: $\left(1+m_{11}\right)\left(1+m_{22}\right)-m_{12} m_{21} \doteq$ $m$ is always positive. Take the inverse transformation of the above equation,

$$
\left[\begin{array}{l}
\frac{\partial X_{\text {new }}}{\partial t} \\
\frac{\partial Y_{\text {new }}}{\partial t}
\end{array}\right]=Q\left[\begin{array}{l}
\frac{\partial X_{\text {old }}}{\partial t} \\
\frac{\partial Y_{\text {old }}}{\partial t}
\end{array}\right], \quad \text { where } \quad Q=\frac{1}{m}\left[\begin{array}{cc}
1+m_{22} & -m_{12} \\
-m_{21} & 1+m_{11}
\end{array}\right]
$$

The jacobian matrix of the original system (10) at $P_{0}$ is

$$
J_{\text {old }}\left(P_{0}\right)=\left[\begin{array}{cc}
F_{X}+\varphi_{X} & F_{Y}+\varphi_{Y} \\
G_{X}+\psi_{X} & G_{Y}+\psi_{Y}
\end{array}\right]_{P_{0}},
$$

and in the jacobian matrix of system (12), the elements at $P_{0}$ is shown as

$$
\begin{aligned}
& j_{11}^{\text {new }}=\left.\left[\frac{\partial\left(\frac{1+m_{22}}{m}\right)}{\partial X}(F+\varphi)+\left(\frac{1+m_{22}}{m}\right)\left(F_{X}+\varphi_{X}\right)+\frac{\partial\left(\frac{-m_{12}}{m}\right)}{\partial X}(G+\psi)+\left(\frac{-m_{12}}{m}\right)\left(G_{X}+\psi_{X}\right)\right]\right|_{P_{0}}, \\
& j_{12}^{\text {new }}=\left.\left[\frac{\partial\left(\frac{1+m_{22}}{m}\right)}{\partial Y}(F+\varphi)+\left(\frac{1+m_{22}}{m}\right)\left(F_{Y}+\varphi_{Y}\right)+\frac{\partial\left(\frac{-m_{12}}{m}\right)}{\partial Y}(G+\psi)+\left(\frac{-m_{12}}{m}\right)\left(G_{Y}+\psi_{Y}\right)\right]\right|_{P_{0}}, \\
& j_{21}^{\text {new }}=\left.\left[\frac{\partial\left(\frac{-m_{21}}{m}\right)}{\partial X}(F+\varphi)+\left(\frac{-m_{21}}{m}\right)\left(F_{X}+\varphi_{X}\right)+\frac{\partial\left(\frac{1+m_{11}}{m}\right)}{\partial X}(G+\psi)+\left(\frac{1+m_{11}}{m}\right)\left(G_{X}+\psi_{X}\right)\right]\right|_{P_{0}}, \\
& j_{22}^{\text {new }}=\left.\left[\frac{\partial\left(\frac{-m_{21}}{m}\right)}{\partial Y}(F+\varphi)+\left(\frac{-m_{21}}{m}\right)\left(F_{Y}+\varphi_{Y}\right)+\frac{\partial\left(\frac{1+m_{11}}{m}\right)}{\partial Y}(G+\psi)+\left(\frac{1+m_{11}}{m}\right)\left(G_{Y}+\psi_{Y}\right)\right]\right|_{P_{0}} .
\end{aligned}
$$


Because $P_{0}$ is the equilibrium point, $(\varphi+F) P_{0}=0,(\psi+G)_{P_{0}}=0$. Therefore

$$
\begin{aligned}
J_{\text {new }}\left(P_{0}\right) & =\left[\begin{array}{cc}
\frac{\left(1+m_{22}\right)\left(F_{X}+\varphi_{X}\right)-m_{12}\left(G_{X}+\psi_{X}\right)}{m} & \frac{\left(1+m_{22}\right)\left(F_{Y}+\varphi_{Y}\right)-m_{12}\left(G_{Y}+\psi_{Y}\right)}{m} \\
\frac{-m_{21}\left(F_{X}+\varphi_{X}\right)+\left(1+m_{11}\right)\left(G_{X}+\psi_{X}\right)}{m} & \frac{-m_{21}\left(F_{Y}+\varphi_{Y}\right)+\left(1+m_{11}\right)\left(G_{Y}+\psi_{Y}\right)}{m}
\end{array}\right]_{P_{0}} \\
& =\frac{1}{\left.m\right|_{P_{0}}}\left[\begin{array}{cc}
1+m_{22} & -m_{12} \\
-m_{21} & 1+m_{11}
\end{array}\right]_{P_{0}}\left[\begin{array}{cc}
F_{X}+\varphi_{X} & F_{Y}+\varphi_{Y} \\
G_{X}+\psi_{X} & G_{Y}+\psi_{Y}
\end{array}\right]_{P_{0}} \\
& =Q\left(P_{0}\right) J_{\text {old }}\left(P_{0}\right) .
\end{aligned}
$$

From the expansion of $\left(\frac{\partial X_{\text {new }}}{\partial t}, \frac{\partial Y_{\text {new }}}{\partial t}\right)^{\prime}=Q\left(\frac{\partial X_{\text {old }}}{\partial t}, \frac{\partial Y_{\text {old }}}{\partial t}\right)^{\prime}$, there is obviously a conclusion: $D_{\text {new }}\left(P_{0}\right)=Q\left(P_{0}\right) D$. Further, we have

$$
H_{\text {new }}(k)=J_{\text {new }}\left(P_{0}\right)+k^{2} D_{\text {new }}\left(P_{0}\right)=Q\left(P_{0}\right)\left(J\left(P_{0}\right)+k^{2} D\right)=Q\left(P_{0}\right) H_{\text {old }}(k) .
$$

This completes the proof.

The above theorem gives the relation between linear approximation system with delay term and the system without delay when $\tau$ is small, which provides a more convenient method to study the stability of equilibrium point by linearizing the time delay term. By solving the transformation matrix $\mathrm{Q}$, key information such as jacobian matrix and characteristic equation of the new system can be obtained quickly. The Turing instability analysis of the approximate system (8), (9) are further given based on Theorem 1.

- For system (8), transformation matrix $Q_{1}$ can be figured out:

$$
Q_{1}=\left[\begin{array}{cc}
1 & 0 \\
0 & \frac{1}{1-2 \tau_{1} \mu I}
\end{array}\right] .
$$

Since the time delay is small and the system is bounded near $P$, we may assume that: $2 \mu I^{*} \tau_{1}<1$, which ensures that $\operatorname{det}\left(Q_{1}\right)$ is positive. According to Theorem 1, we have $J_{1}(P)=Q_{1}(P) J(P), D_{1}(P)=Q_{1}(P) D, H_{1}(K)=Q_{1}(P) H(K)$, where $J_{1}(P)$ and $D_{1}(P)$ respectively represent the Jacobian matrix and diffusion coefficient matrix of system (8), and $H_{1}(K) \triangleq J_{1}(P)-k^{2} D_{1}(P)$. Further, the positive and negative properties of $J_{1}$ and $H_{1}$ 's determinants and traces of the new system are analyzed in order to obtain the conditions of Turing pattern. The following conclusions can be reached:

$$
\begin{aligned}
& \operatorname{tr}\left(J_{1}(P)\right)=S^{*}\left(2 \beta^{2} \frac{1}{\mu}-K-A\right)+2 A K-\frac{\beta S^{*}}{1-2 \tau_{1} \mu I^{*}}<\operatorname{tr}(J(P)), \\
& \operatorname{tr}\left(H_{1}(k)\right)=\operatorname{tr}\left(J_{1}(P)\right)-k^{2}\left(d_{11}+\frac{d_{22}}{1-2 \tau_{1} \mu I^{*}}\right)<\operatorname{tr}(H(k)), \\
& \operatorname{det}\left(J_{1}(P)\right)=\frac{1}{1-2 \tau_{1} \mu I^{*}} \operatorname{det}(J(P)), \\
& \operatorname{det}\left(H_{1}(k)\right)=\frac{1}{1-2 \tau_{1} \mu I^{*}} \operatorname{det}(H(k)) .
\end{aligned}
$$

Observe the necessary conditions for the existence of Turing bifurcation for system (6) in the previous section. Under the assumption of $\beta<\mu$, we have $\operatorname{tr}(J(P))<0, \operatorname{tr}(H(P))<0$, therefore the new system satisfies $\operatorname{tr}\left(J_{1}(P)\right)<0, \operatorname{tr}\left(H_{1}(P)\right)<0$. At the same time, since $\operatorname{det}\left(J_{1}(P)\right)$ has the same sign as $\operatorname{det}(J(P))$, so $\operatorname{do} \operatorname{det}\left(H_{1}(P)\right)$ and $\operatorname{det}(H(P))$, it can be found that the necessary conditions for the occurrence of Turing instability of the system given by the method in previous section is also necessary for Turing bifurcation of the system (8) to occur when the time delay is small. The conditions for the existence of diffusion-driven Turing instability of system (8) are

$$
\theta<0, \quad 4 \eta \Delta_{0}<\theta^{2}, \quad \tau_{1}<\frac{1}{2 \mu I^{*}} .
$$

It is worth noting that although this conclusion is concise and effective, it is not universal. The main reason is that the time delay approximation matrix is a simple positive diagonal matrix, and the element in the lower right corner amplifies the negative term in the $\operatorname{tr}(J)$, so that the trace of $J_{1}$ and $H_{1}$ is always negative under the original condition. In the discussion of the correlation conditions of determinant of $J_{1}$ and $H_{1}$, the conditions controlling the value of $\operatorname{det}(J)$ and $\operatorname{det}(H)$ can also control the positive and negative properties of $\operatorname{det}\left(J_{1}\right)$ and $\operatorname{det}\left(H_{1}\right)$, because they have the same sign as the original system. 
- For system (9), transformation matrix $Q_{2}$ can be figured out:

$$
Q_{2}=\frac{1}{1+\tau_{2} \beta S-\tau \beta I}\left[\begin{array}{cc}
1+\tau_{2} \beta S & \tau_{2} \beta S \\
-\tau_{2} \beta I & 1-\tau_{2} \beta I
\end{array}\right]
$$

In the vicinity of the equilibrium point $\mathrm{P}$, based on the fact that $\beta S^{*}=\mu I^{*}$, and the hypothesis that $\beta<\mu$, $\operatorname{det}\left(Q_{2}\right)=$ $\frac{1}{1+\tau_{2} \beta S-\tau_{2} \beta I}$ is greater than 0 . Let's label the matrix associated with system (9) with subscript 2 . Similar to the above process, we draw the following conclusions directly:

$$
\begin{aligned}
& \operatorname{tr}\left(J_{2}(P)\right)=\frac{1}{1+\tau_{2}(\mu-\beta) I^{*}}\left[\operatorname{tr}(J(P))+\tau_{2} \beta S^{*}\left(S^{*}\left(\frac{5 \beta^{2}}{\mu}-K-A\right)+2 A K\right)\right], \\
& \operatorname{tr}\left(H_{2}(k)\right)=\operatorname{tr}\left(J_{2}(P)\right)-\frac{k^{2}}{1+\tau_{2}(\mu-\beta) I^{*}}\left[d_{11}+d_{22}+\tau_{2} I^{*}\left(\mu d_{21}+\mu d_{11}-\beta d_{12}-\beta d_{22}\right)\right], \\
& \operatorname{det}\left(J_{2}(P)\right)=\frac{1}{1+\tau_{2}(\mu-\beta) I^{*}} \operatorname{det}(J(P)), \\
& \operatorname{det}\left(H_{2}(k)\right)=\frac{1}{1+\tau_{2}(\mu-\beta) I^{*}} \operatorname{det}(H(k)) .
\end{aligned}
$$

Unlike the time-delay approximation system discussed in the previous part, although the determinant of $J_{2}$ and $H_{2}$ are still the same sign as the original system, the trace of $J_{2}$ and $\mathrm{H}_{2}$ cannot be guaranteed to be less than 0 under the constraint of existing conditions. In order to make the real part of the eigenvalue under the influence of no diffusion negative, let $\operatorname{tr}\left(J_{2}\right)<0$, the following constraints can be obtained:

$$
\tau_{2}<\frac{-\operatorname{tr}(J(P))}{\beta S^{*}\left[S^{*}\left(5 \beta^{2} \frac{1}{\mu}-K-A\right)+2 A K\right]}, \quad \text { if } \quad \beta S^{*}\left[S^{*}\left(5 \beta^{2} \frac{1}{\mu}-K-A\right)+2 A K\right]>0 .
$$

If $\beta S^{*}\left[S^{*}\left(5 \beta^{2} \frac{1}{\mu}-K-A\right)+2 A K\right] \leq 0$, it is always true that $\operatorname{tr}\left(J_{2}\right)<0$. In this case, when the parameters satisfy the existence of $k$ such that $\operatorname{tr}(H(k))>0$, the conclusion that $P$ is unstable under diffusion-driven can also be deduced. The instability conditions are as follows:

$$
\left\{\begin{array}{l}
\tau_{2}>\frac{d_{11}+d_{21}}{I^{*}\left(\beta d_{12}+\beta d_{22}-\mu d_{21}-\mu d_{11}\right)} \\
0<\beta d_{12}+\beta d_{22}-\mu d_{21}-\mu d_{11}
\end{array}\right.
$$

When the original system (3) does not show Turing bifurcation phenomenon near $P, \operatorname{namely} \operatorname{tr}(J)<0, \operatorname{det}(J)>0, \operatorname{tr}(H)<$ $0, \operatorname{det}(H)>0, \operatorname{det}(H 2)>0$, but in system (9) to meet the above conditions $\operatorname{tr}(H 2)>0$. Such instability can be called the Turing bifurcation phenomenon co-driven by diffusion and time delay. As shown in Fig.1, the threshold curve of $\tau_{2}$ induced instability in the approximate system is given, which varies with $d_{12}$. This instability condition does not exist in the model analyzed in the previous part. When added the $\operatorname{tr}(H 2)<0$, similar to the previous model (8), the determinant is the same sign as the original model. When $\tau_{2}$ is small enough, the necessary condition for the existence of Turing bifurcation in the original model is also the necessary condition of model (9).

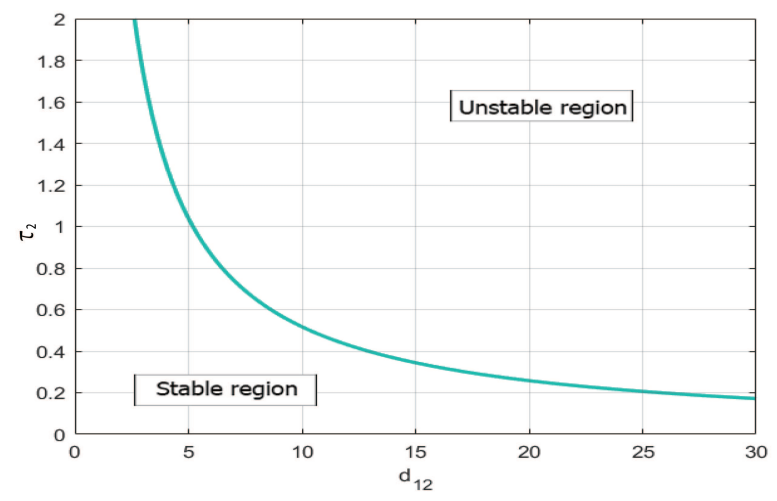

FIG. 1. Threshold curve of Turing instability induced by $\tau_{2}$ changing with $d_{12}$ in the approximation system of system (9). Other parameters are set as follows: $K=1, A=0.1, \beta=0.46, \mu=0.5, d_{11}=0.5, d_{22}=0.5, d_{21}=0$. 


\section{System of period coefficient diffusion}

In many reaction-diffusion systems, it is not always reasonable and effective to assume that the diffusion coefficient of the subject is constant. In fact, there are many diffusion processes in nature whose intensity is closely related to the time of diffusion. In the actual background of rumor propagation, the diffusion coefficient of the crowd in the information network space may also be considered as periodic.

We assume that the diffusion coefficient and time satisfy the following periodic relation: $D(t)=d+d^{\prime} \sin (\omega t+\phi)$, where $d$, $d^{\prime}, \omega$ and $\phi$ are constants. The periodic diffusion coefficient system can be written as

$$
\left\{\begin{array}{l}
\frac{\partial S}{\partial t}=\left[d_{11}+d^{\prime}{ }_{11} \sin \left(\omega t+\phi_{11}\right)\right] \Delta S+\left[d_{12}+d^{\prime}{ }_{12} \sin \left(\omega t+\phi_{12}\right)\right] \Delta I+S(K-S)(S-A)-\beta S I \\
\frac{\partial I}{\partial t}=\left[d_{21}+d^{\prime}{ }_{21} \sin \left(\omega t+\phi_{21}\right)\right] \Delta S+\left[d_{22}+d^{\prime}{ }_{22} \sin \left(\omega t+\phi_{22}\right)\right] \Delta I+\beta S I-\mu I^{2}
\end{array}\right.
$$

In this part, we expand the superposition perturbation near the rumor-spreading equilibrium point in the Fourier space into the system with linearization and derive the first-order approximation system on a single time dimension based no Ref. ${ }^{29}$. Then the stability of the system is further analyzed by using Floquet theory ${ }^{30}$. The derivation process of the first-order system is highly overlapped with the the derivation process of characteristic equation of the diffusion system. The first-order system is

$$
\vec{\delta}_{t}=U_{\omega}(k, t) \vec{\delta}
$$

where $U_{\omega}(k, t) \triangleq J-k^{2} D_{\omega}(\mathrm{t}), \vec{\delta}=\left(\delta_{1}, \delta_{2}\right)^{\prime}$ is perturbation, $k$ is wave number and $D_{\omega}(\mathrm{t})$ is the diffusion coefficient matrix with $\omega$ as the period. The stability of the system can be determined by $U_{\omega}(t)$.

According to Floquet's theory, there must be a solution that satisfies the following form:

$$
\vec{\delta}\left(t+\frac{2 \pi}{\omega}\right)=\varepsilon \cdot \vec{\delta}(t)
$$

where $\varepsilon$ can be obtained by calculating the characteristic roots of $E$, which refers to the transformation matrix of the fundamental solution matrix $\Phi(t)$ of system (15) satisfying $\Phi\left(t+\frac{2 \pi}{\omega}\right)=E \cdot \Phi(t)$.

By observing the form of the solution, it is obvious that when $|\varepsilon|>1$, the system has an unbounded solution, when $|\varepsilon|<1$, the system has a stable solution, when $|\varepsilon|=1$, the system has a periodic solution. Further, when $\varepsilon=1, \vec{\delta}\left(t+\frac{2 \pi}{\omega}\right)=\vec{\delta}(t)$, the minimum period of the periodic solution is $\frac{2 \pi}{\omega}$, and the minimum period is $\frac{4 \pi}{\omega}$, when $\varepsilon=-1, \vec{\delta}\left(t+\frac{4 \pi}{\omega}\right)=-\vec{\delta}\left(t+\frac{2 \pi}{\omega}\right)=\vec{\delta}(t)$. It is effective to calculate the multiplicators in judging the stability of the solution. However, in most cases, it is difficult to calculate the fundamental solution matrix of nonautonomous differential equations, so is this system. So it's almost impossible to figure out the characteristic multiplier by solving the fundamental solution matrix of system (15). Therefore we need a new method to estimate the range of $\varepsilon$.

The multiplicators $\varepsilon_{1}$ and $\varepsilon_{2}$ can be expressed as the roots of

$$
\varepsilon^{2}-u(k, \omega) \varepsilon+c=0
$$

where the form of $u(k, \omega)$ is difficult to determine, which is related to $U_{\omega}(k, t)$. But fortunately, $c$ can be given by the following formula:

$$
c=\varepsilon_{1} \varepsilon_{2}=\exp \left(\int_{0}^{2 \pi} \operatorname{tr}\left(U_{\omega}(k, t)\right) d t\right)
$$

Meanwhile $0<c<1$, with $\int_{0}^{2 \pi} \operatorname{tr}\left(U_{\omega}(k, t)\right) d t=\frac{2 \pi}{\omega}\left(\operatorname{tr}(J)-k^{2}\left(d_{11}+d_{22}\right)\right)<0$. Multiplicators can be represented by $u$ and $c$ : $\varepsilon_{1}=\frac{1}{2}\left(u+\sqrt{u^{2}-4 c}\right), \varepsilon_{2}=\frac{1}{2}\left(u-\sqrt{u^{2}-4 c}\right)$. We can do the following analysis for different values of $u$ :

(1) $|u|>1+c$. In this caes, $u^{2}-4 c>0$, and $\varepsilon_{1}$, $\varepsilon_{2}$ are both negative real numbers with $\varepsilon_{2}<-1<-c<\varepsilon_{1}<0$, or $0<\varepsilon_{2}<c<1<\varepsilon_{1}$. Equation (15) has an unbounded solution.

(2) $|u|=1+c$. In this caes, $u^{2}-4 c>0$. When $u<0, \varepsilon_{2}=-1$, and system (15) has a solution with period $\frac{4 \pi}{\omega}$. When $u>0$, there is a solution with period $\frac{2 \pi}{\omega}$ for $\varepsilon_{1}=1$.

(3) $2 \sqrt{c} \leq|u|<1+c$. In this caes, $u^{2}-4 c \geq 0$. $\varepsilon_{1}$ and $\varepsilon_{2}$ both are real numbers with the same sign whose modulus is less than 1 . The solution to system (15) is stable. 
(4) $|u|<2 \sqrt{c}$. In this caes, $u^{2}-4 c<0$, and $\varepsilon_{1}, \varepsilon_{2}$ are conjugate with non-zero imaginary parts. System (15) has the general solution in the following form:

$$
\vec{\delta}(t)=c_{1} \exp \left(\frac{2 \pi \operatorname{lnc}}{\omega} t+i v t\right)\left(\begin{array}{c}
p_{11}(t) \\
p_{21}(t)
\end{array}\right)+c_{2} \exp \left(\frac{2 \pi \operatorname{lnc}}{\omega} t-i v t\right)\left(\begin{array}{c}
p_{12}(t) \\
p_{22}(t)
\end{array}\right),
$$

$\left|\varepsilon_{1}\right|<1,\left|\varepsilon_{2}\right|<1, c_{1}, c_{2}$ are arbitrary constants, $p_{11}(t), p_{12}(t), p_{21}(t)$ and $p_{22}(t)$ are periodic function. The solution is oscillating, but not generally periodic.

The region where the above parameters make the solutions all bounded with no period is defined as the stable parametric region, the region where the system has unbounded solutions is defined as the unstable parametric region, and the region where the equation has periodic solutions is defined as the periodic region. Fig.2(a) intuitively shows the various regional attributes of $u-c$ in the above 4 cases.

It can be found that the periodic region divides the parameter stable and unstable regions of system (15). In order to obtain the instability of system (15), we consider using the numerical solution method to solve the condition that the parameters satisfy when the periodic solution exists.

Fourier expansion of $\vec{\delta}$ with period of $\frac{4 \pi}{\omega}$ can be obtained by

$$
\vec{\delta}=\left(\begin{array}{l}
\delta_{1} \\
\delta_{2}
\end{array}\right)=\sum_{n=-\infty}^{n=+\infty}\left(\begin{array}{l}
A_{n} e^{\left(a_{0}+\frac{i n \omega}{2}\right) t} \\
B_{n} e^{\left(b_{0}+\frac{i n \omega}{2}\right) t}
\end{array}\right)
$$

Let $a_{0}+\frac{i n \omega}{2}:=a_{n}, b_{0}+\frac{i n \omega}{2}:=b_{n}$. Insert expansion into system $(15)$, make $\sin (x)=\frac{1}{2 i}\left(e^{i x}-e^{-i x}\right)$ and we can obtain

$$
\begin{aligned}
& \sum_{-\infty}^{+\infty} e^{a_{n} t}\left[\left(a_{n}-j_{11}+k^{2} d_{11}\right) A_{n}+\frac{1}{2 i} d^{\prime}{ }_{11} k^{2} e^{i \phi_{11}} A_{n-2}-\frac{1}{2 i} d^{\prime}{ }_{11} k^{2} e^{-i \phi_{11}} A_{n+2}\right] \\
+ & \sum_{-\infty}^{+\infty} e^{b_{n} t}\left[\left(-j_{12}+k^{2} d_{12}\right) B_{n}+\frac{1}{2 i} d^{\prime}{ }_{12} k^{2} e^{i \phi_{12}} B_{n-2}-\frac{1}{2 i} d^{\prime}{ }_{12} k^{2} e^{-i \phi_{12}} B_{n+2}\right]=0 \\
& \sum_{-\infty}^{+\infty} e^{a_{n} t}\left[\left(-j_{21}+k^{2} d_{21}\right) A_{n}+\frac{1}{2 i} d^{\prime}{ }_{21} k^{2} e^{i \phi_{21}} A_{n-2}-\frac{1}{2 i} d^{\prime}{ }_{21} k^{2} e^{-i \phi_{21}} A_{n+2}\right] \\
+ & \sum_{-\infty}^{+\infty} e^{b_{n} t}\left[\left(b_{n}-j_{22}+k^{2} d_{22}\right) B_{n}+\frac{1}{2 i} d^{\prime}{ }_{22} k^{2} e^{i \phi_{22}} B_{n-2}-\frac{1}{2 i} d^{\prime}{ }_{22} k^{2} e^{-i \phi_{22}} B_{n+2}\right]=0
\end{aligned}
$$

The formula is true for any $t$, and let $t=0$, then

$$
\begin{aligned}
& \left(a_{n}-j_{11}+k^{2} d_{11}\right) A_{n}+\frac{1}{2 i} d^{\prime}{ }_{11} k^{2} e^{i \phi_{11}} A_{n-2}-\frac{1}{2 i} d^{\prime}{ }_{11} k^{2} e^{-i \phi_{11}} A_{n+2} \\
+ & \left(-j_{12}+k^{2} d_{12}\right) B_{n}+\frac{1}{2 i} d^{\prime}{ }_{12} k^{2} e^{i \phi_{12}} B_{n-2}-\frac{1}{2 i} d^{\prime}{ }_{12} k^{2} e^{-i \phi_{12}} B_{n+2}=0 \\
& \left(-j_{21}+k^{2} d_{21}\right) A_{n}+\frac{1}{2 i} d^{\prime}{ }_{21} k^{2} e^{i \phi_{21}} A_{n-2}-\frac{1}{2 i} d^{\prime}{ }_{21} k^{2} e^{-i \phi_{21}} A_{n+2} \\
+ & \left(b_{n}-j_{22}+k^{2} d_{22}\right) B_{n}+\frac{1}{2 i} d^{\prime}{ }_{22} k^{2} e^{i \phi_{22}} B_{n-2}-\frac{1}{2 i} d^{\prime}{ }_{22} k^{2} e^{-i \phi_{22}} B_{n+2}=0
\end{aligned}
$$

The formula is an infinite set of homogeneous linear equations.

Remark 1. When system (15) has a solution with a period of $\frac{2 \pi}{\omega}$, it is required that $A_{n}$ and $B_{n}$ have solutions that are not all zero when $n$ only takes odd numders. This part is consistent with the document ${ }^{29,31-33}$.

Remark 2. Similar to above, when system (15) has a solution with a period of $\frac{4 \pi}{\omega}$, it is required that $A_{n}$ and $B_{n}$ have solutions that are not all zero. Given the fact that this situation includes the solution with a period of $\frac{2 \pi}{\omega}$, to get solutions periodic of period $\frac{4 \pi}{\omega}$ instead of $\frac{2 \pi}{\omega}$, conditions that there are non-zero solutions for some $A_{n}$ and $B_{n}$ must be satisfied when $n$ takes even numbers. Therefore, we only need to consider whether the corresponding $A_{n}$ and $B_{n}$ are all nil when $n$ is only even to determine the existence condition of the solution with a minimum period of $\frac{4 \pi}{\omega}$.

Since it is a homogeneous system of equations, the condition for the above two cases is that the infinite determinant formed by the coefficients of the corresponding $A_{n}$ and $B_{n}$, known as a Hill determinant, is nil. For the convergence of the Hill determinant, 
each equation is divided by $\left(1+n^{2}\right)$. For simplicity, make the following definition:

$$
\begin{array}{rlrl}
\gamma_{n}^{(11)} & =\frac{a_{n}-j_{11}+k^{2} d_{11}}{1+n^{2}}, & \gamma_{n}^{(12)}=\frac{-j_{12}+k^{2} d_{12}}{1+n^{2}}, \\
\gamma_{n}^{(21)}=\frac{-j_{21}+k^{2} d_{21}}{1+n^{2}}, & \gamma_{n}^{(22)}=\frac{b_{n}-j_{22}+k^{2} d_{22}}{1+n^{2}}, \\
\xi_{n}^{(11)}=\frac{d^{\prime}{ }_{11} k^{2} e^{i \phi_{11}}}{2 i\left(1+n^{2}\right)}, & \xi_{n}^{(12)}=\frac{d^{\prime}{ }_{12} k^{2} e^{i \phi_{12}}}{2 i\left(1+n^{2}\right)}, \\
\xi_{n}^{(21)}=\frac{d^{\prime}{ }_{21} k^{2} e^{i \phi_{21}}}{2 i\left(1+n^{2}\right)}, & \xi_{n}^{(22)}=\frac{d^{\prime}{ }_{22} k^{2} e^{i \phi_{22}}}{2 i\left(1+n^{2}\right)}, \\
\zeta_{n}^{(11)}=-\frac{d^{\prime}{ }_{11} k^{2} e^{-i \phi_{11}}}{2 i\left(1+n^{2}\right)}, & \zeta_{n}^{(12)}=-\frac{d^{\prime}{ }_{12} k^{2} e^{-i \phi_{12}}}{2 i\left(1+n^{2}\right)}, \\
\zeta_{n}^{(21)}=-\frac{d^{\prime}{ }_{21} k^{2} e^{-i \phi_{21}}}{2 i\left(1+n^{2}\right)}, & \zeta_{n}^{(22)}=-\frac{d^{\prime}{ }_{22} k^{2} e^{-i \phi_{22}}}{2 i\left(1+n^{2}\right)} .
\end{array}
$$

The 6 order determinant of Hill determinant when $n$ takes odd number (system has a periodic solution of $\frac{2 \pi}{\omega}$ ) is given by

$$
H_{6 \times 6}^{o d d}=\left|\begin{array}{cccccc}
\gamma_{-2}^{(11)} & \gamma_{-2}^{(12)} & \zeta_{-2}^{(11)} & \zeta_{-2}^{(12)} & 0 & 0 \\
\gamma_{-2}^{(21)} & \gamma_{-2}^{(22)} & \zeta_{-2}^{(21)} & \zeta_{-2}^{(22)} & 0 & 0 \\
\xi_{0}^{(11)} & \xi_{0}^{(12)} & \gamma_{0}^{(11)} & \gamma_{0}^{(12)} & \zeta_{0}^{(11)} & \zeta_{0}^{(12)} \\
\xi_{0}^{(21)} & \xi_{0}^{(22)} & \gamma_{0}^{(21)} & \gamma_{0}^{(22)} & \zeta_{0}^{(21)} & \zeta_{0}^{(22)} \\
0 & 0 & \xi_{2}^{(11)} & \xi_{2}^{(12)} & \gamma_{2}^{(11)} & \gamma_{2}^{(12)} \\
0 & 0 & \xi_{2}^{(21)} & \xi_{2}^{(22)} & \gamma_{2}^{(21)} & \gamma_{2}^{(22)}
\end{array}\right|
$$

and the 8 order determinant of Hill determinant when $n$ takes even number (system has a periodic solution of $\frac{4 \pi}{\omega}$ ) is given by

$$
H_{8 \times 8}^{\text {even }}=\left|\begin{array}{cccccccc}
\gamma_{-3}^{(11)} & \gamma_{-3}^{(12)} & \zeta_{-3}^{(11)} & \zeta_{-3}^{(12)} & 0 & 0 & 0 & 0 \\
\gamma_{-3}^{(21)} & \gamma_{-3}^{(22)} & \zeta_{-3}^{(21)} & \zeta_{-3}^{(22)} & 0 & 0 & 0 & 0 \\
\xi_{-1}^{(11)} & \xi_{-1}^{(12)} & \gamma_{-1}^{(11)} & \gamma_{-1}^{(12)} & \zeta_{-1}^{(11)} & \zeta_{-1}^{(12)} & 0 & 0 \\
\xi_{-1}^{(21)} & \xi_{-1}^{(22)} & \gamma_{-1}^{(21)} & \gamma_{-1}^{(22)} & \zeta_{-1}^{(21)} & \zeta_{-1}^{(22)} & 0 & 0 \\
0 & 0 & \xi_{1}^{(11)} & \xi_{1}^{(12)} & \gamma_{1}^{(11)} & \gamma_{1}^{(12)} & \zeta_{1}^{(11)} & \zeta_{1}^{(12)} \\
0 & 0 & \xi_{1}^{(21)} & \xi_{1}^{(22)} & \gamma_{1}^{(21)} & \gamma_{1}^{(22)} & \zeta_{1}^{(21)} & \zeta_{1}^{(22)} \\
0 & 0 & 0 & 0 & \xi_{2}^{(11)} & \xi_{2}^{(12)} & \gamma_{2}^{(11)} & \gamma_{2}^{(12)} \\
0 & 0 & 0 & 0 & \xi_{2}^{(21)} & \xi_{2}^{(22)} & \gamma_{2}^{(21)} & \gamma_{2}^{(22)}
\end{array}\right|
$$

Numerical solution methods such as Newton's method can be used to draw parametric feasible curves of the above two determinants respectively equal to zero. As shown in Fig.2(b), after selecting a set of parameters, the boundary curves of stability and instability of the system based on the changes of $k$ and $\omega$, namely the periodic solution curve, are given. Further, the value of $k$ and $\omega$ marked with red letters is selected in panel (b), and the corresponding variation curves of system disturbance are given by using the four-five-order Range-Kuttle method algorithm in panel (c)-(f) of Fig.2. In Fig.2(c), we give a set of initial values satisfying the perturbation instability, and obtain the solution curve of the system. In Fig.2(d) and 2(e), the parameters fall on the periodic curves, and the disturbance (initial value is set as $(0.1,-0.1)$ ) presents a vibration phenomenon with a period of $\frac{2 \pi}{\omega}$ and $\frac{4 \pi}{\omega}$. In Fig.2(f), the parameter is in the stable region, and the solution curve with the initial value of $(0.1,-0.1)$ finally converges to zero.

In order to appear Turing instability, we require the system to be stable when $k=0$ and unstable when $k>0$, so we can try to give the expression of the necessary conditions for the existence of Turing pattern in the periodic diffusion coefficient system analogously ${ }^{29}$ : After a set of parameters is selected, an image similar to that shown in Fig.2(b) can be obtained, and $\omega$ can be fixed at the same time. If Turing instability exists in the system, it must satisfy the conditions that when $k=0$, the system falls on the stable region and there is $k>0$ makes the system is in the unstable region. Since $\omega$ satisfies the above necessary conditions in the range shown in Fig.2(b), we can try to obtain Turing pattern of the periodic system under such parameters. It is worth noting that if $d^{\prime}=0$, that is, when there is no periodic term, with the same rest of parameters, the pattern phenomenon of the constant diffusion coefficient system can still be derived. However, in the following numerical simulation, we give an example that Turing pattern exists with period terms but not with $d^{\prime}=0$. 


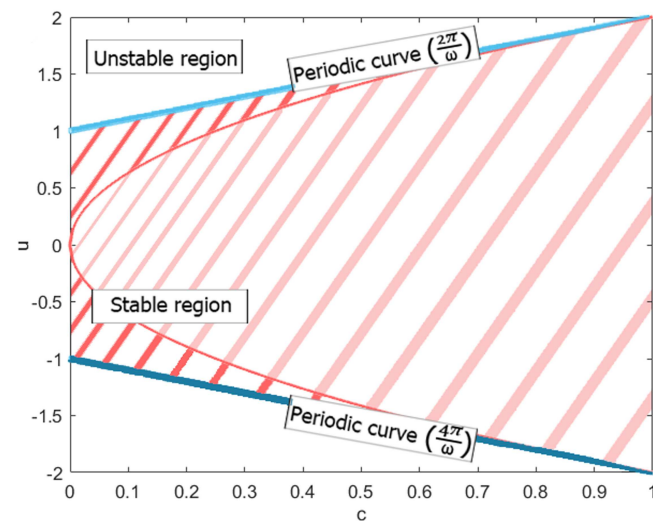

(a)

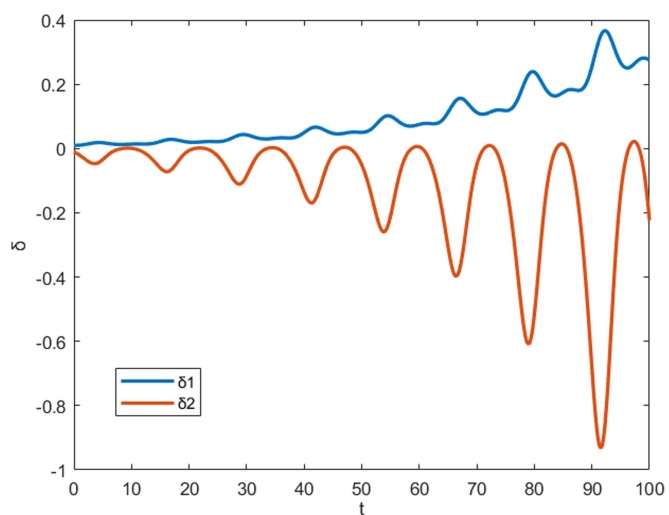

(c)

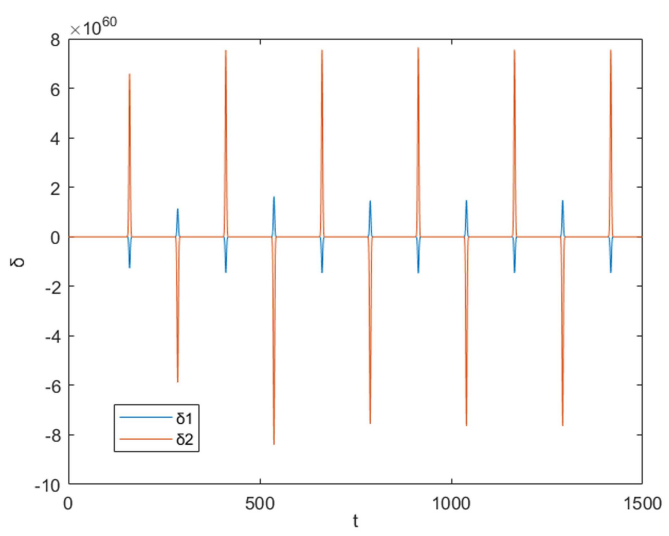

(e)

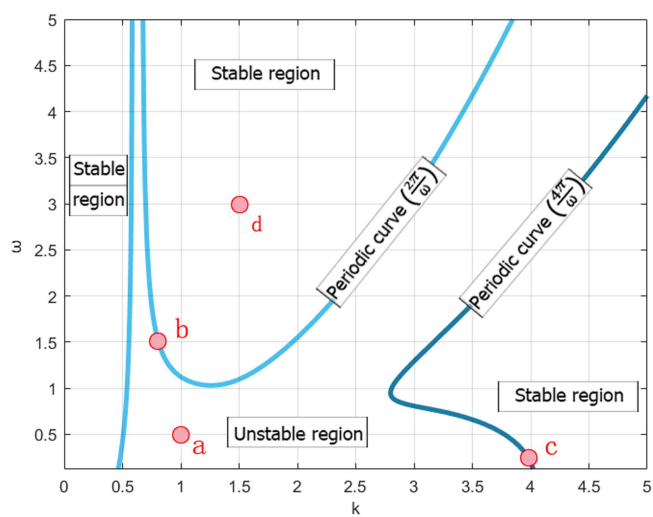

(b)

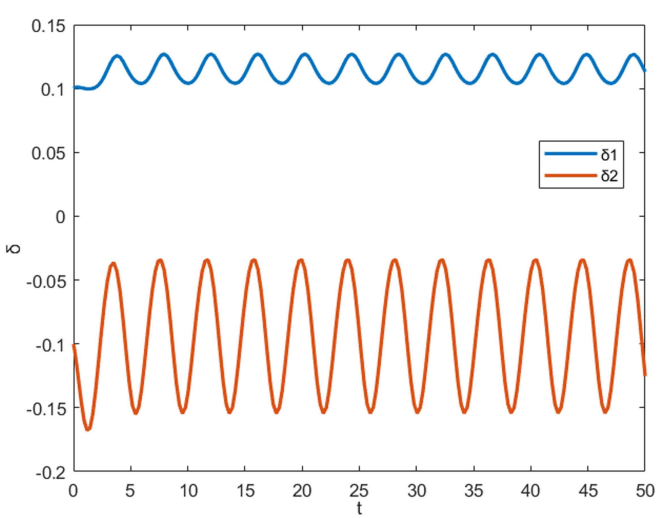

(d)

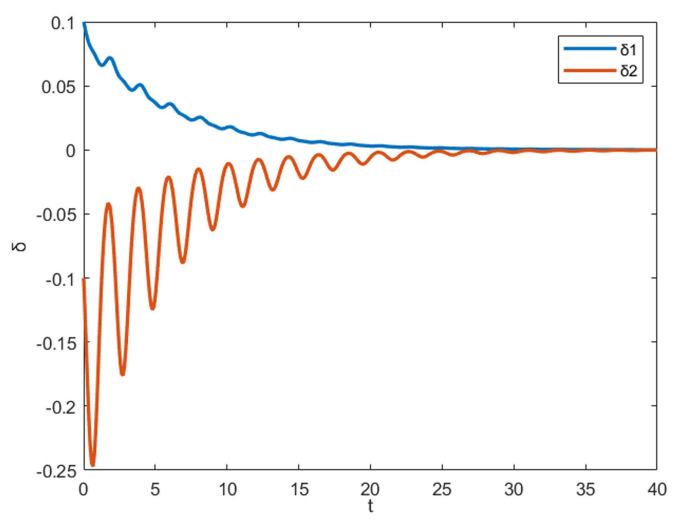

(f)

FIG. 2. Panel (b) shows the state of system (15) under different values of $u$ and $c$. Panel (b) shows the state of system (15) under different values of $k$ and $\omega$. Panel (c)-(f) show some special solution curves whose parameters correspond to the four points $a(k=1, \omega=0.5), b$ $(k=0.8, \omega=1.53), c(k=4, \omega=0.05), d(k=1.5, \omega=3)$ marked in panel (b) respectively. In panel (b)-(f), the other parameters are set as follows: $K=1, A=0.1, \beta=0.46, \mu=0.5, d_{11}=d_{11}^{\prime}=0.5, d_{22}=d_{22}^{\prime}=0.5, d_{12}=d_{12}^{\prime}=0.1, d_{21}=d_{21}^{\prime}=0.965, \phi_{11}=0, \phi_{22}=\frac{4 \pi}{3}$, $\phi_{12}=\frac{\pi}{6}, \phi_{21}=\frac{\pi}{6}, \theta_{1}=0, \theta_{2}=0$.

\section{NUMERICAL SIMULATION}

In this section, we conduct extensive numerical simulation of the spatial patterns of the above mentioned systems, which are all defined in $\Omega \times T$, where $\Omega=[0,100] \times[0,100]$ is a two-dimensional rectangular space, and $T=[0,+\infty)$. In this section, the numerical simulation of process adopting zero flux boundary conditions, numerical integration employs forward euler integral 
method, and central difference scheme are used for the numerical approximation of Laplacian. In the process of iterative, the time step $\Delta t$ takes 0.01 , and the space step $\Delta h$ takes 1 .

In addition, the number of iterations of pattern formations given is not always the same, as detailed after each figure, and the last one of the multiple patterns given by the same system at different points in time evolution can always represents the stable state of the system, that is, the patterns after that will not change. By observing the pattern types of the system under different parameters, it can be found that the pattern types of $S$ and $I$ are always consistent. Therefore, in the following simulation results, we will only give the diagrams of $S$ 's density distribution.

\section{A. Constant diffusion coefficient}

In this part, we simulate the formation of the pattern for system (3) and compare the types of patterns under different values of diffusion coefficients. As shown in Fig.3, there is no cross diffusion $\left(d_{12}=d_{21}=0\right)$. When $t=250$, as shown in Fig.3(a) the system mainly shows strips in space. With the advance of time, cold spots begin to appear, and at this time, cold spots and strips compete with each other, as shown in Fig.3(b). Finally, the spatial distribution of the system evolves into an irregular cold spots with red (high density) as the background, that is, some independent circular regions with low density appear in the space.

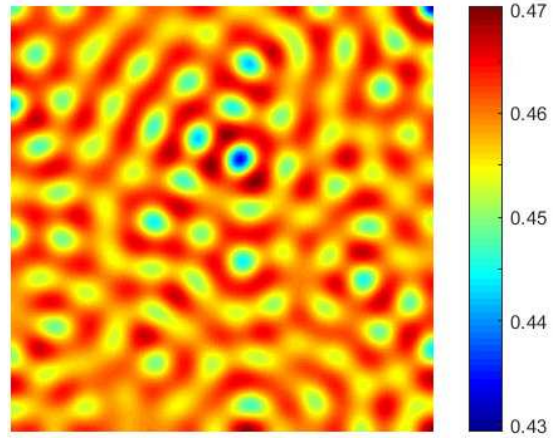

(a)

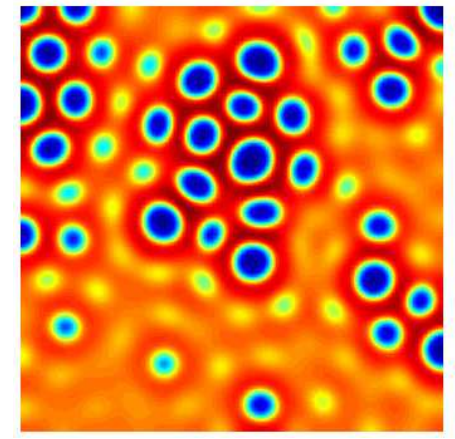

(b)
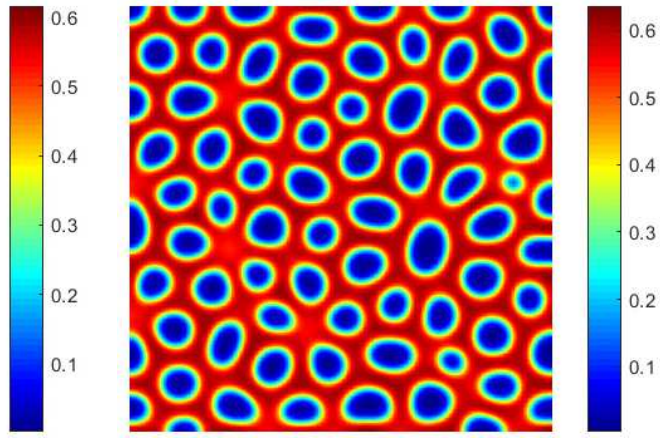

(c)

FIG. 3. In the case that $d_{11}=0.1, d_{22}=2, d_{12}=d_{21}=0$, the evolution process of Turing pattern for system (3) is shown. In panel (a), $t=250$. In panel (b), $t=500$. In panel (c), $t=750$. The other parameters are set as follows: $K=1, A=0.1, \beta=0.46, \mu=0.5$.

When the system is affected by cross-diffusion $\left(d_{12}=0.2, d_{21}=0.4\right)$, the formation time of the stable spot pattern of the system is significantly reduced. When $t=20$, as shown in Fig.4(a), the competition between spots and strips also appears in the pattern, but with the evolution of time, the pattern finally shows the coexistence of cold spots and cold strips. At this time, in the distribution of $S$, some independent regions with low density also appear in the space, but at this time, the shapes of these regions are greatly different.

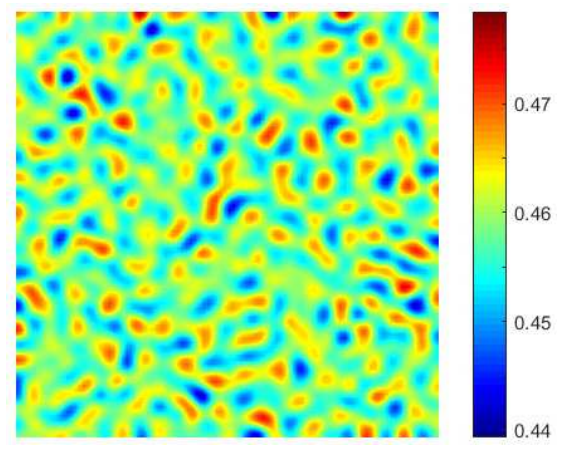

(a)

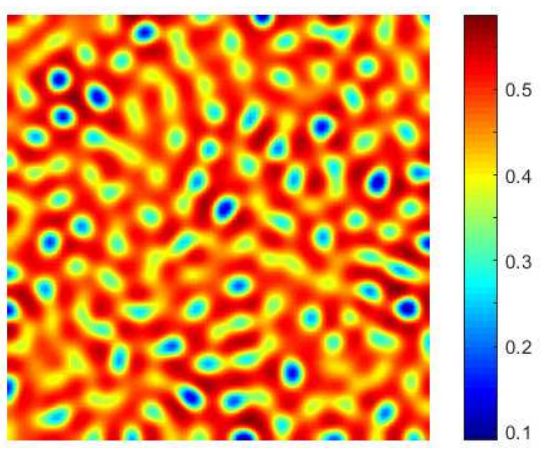

(b)

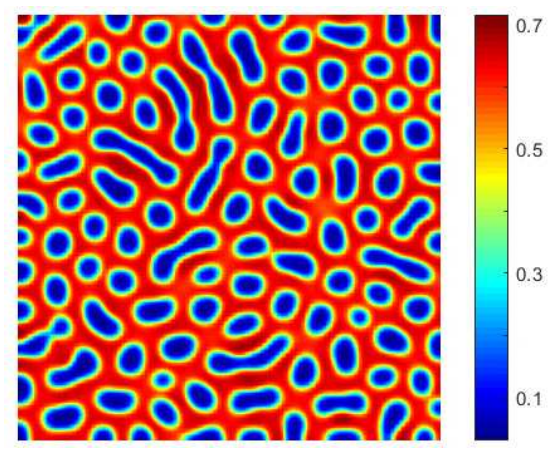

(c)

FIG. 4. In the case that $d_{11}=0.1, d_{22}=2, d_{12}=0.2, d_{21}=0.4$, the evolution process of Turing pattern for system (3) is shown. In panel (a), $t=20$. In panel (b), $t=40$. In panel (c), $t=60$. The other parameters are set as follows: $K=1, A=0.1, \beta=0.46, \mu=0.5$.

In Fig. 3 and Fig.4, the diffusion coefficient $d_{22}$ is much higher than $d_{11}$. In Fig.5, $d_{11}$ and $d_{22}$ are taken as 0.5 , at which time the pattern type changes significantly. In Fig.5(a), the competition between hot spots and hot bars appears, and finally 
the red spots are stable on the blue background, and the distribution of the points is very regular. At this time, a high-density circular independent region appears in the background of low density, and the distribution of $S$ in space appears aggregation phenomenon.

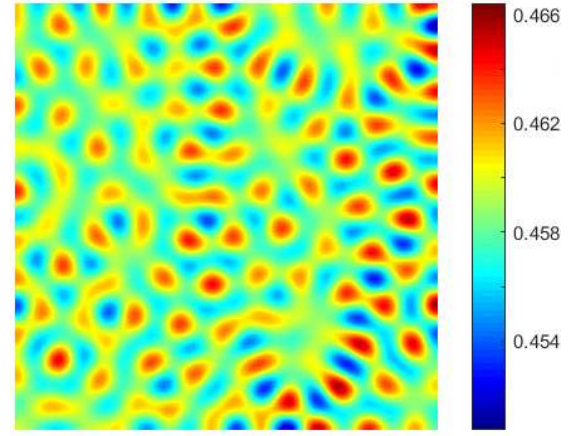

(a)

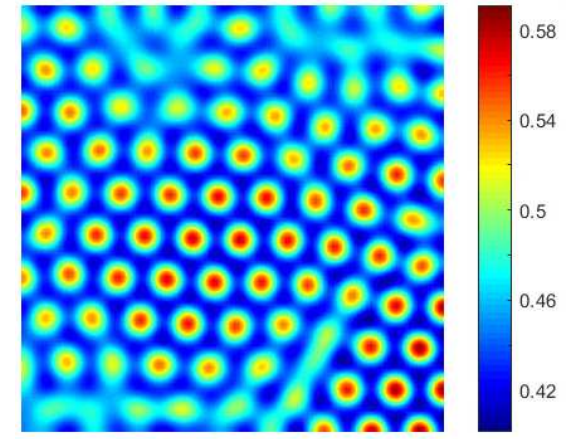

(b)

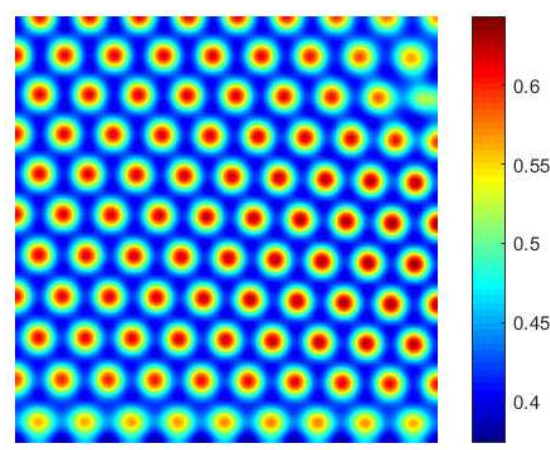

(c)

FIG. 5. In the case that $d_{11}=0.5, d_{22}=0.5, d_{12}=0.1, d_{21}=0.965$, the evolution process of Turing pattern for system (2) is shown. In panel (a), $t=2000$. In panel (b), $t=4000$. In panel (c), $t=6000$. The other parameters are set as follows: $K=1, A=0.1, \beta=0.46, \mu=0.5$.

\section{B. Pattern driven by time delay}

In the previous Fig.1, we have given the critical curve of the approximate system with time delay driving its instability as $d_{12}$ changes. In the unstable parameter region, $\tau_{2}=0.25$ and $d_{12}=24$ are taken, and the stereoscopic pattern of system (14) when $t=1000$ is given in panel (b). For comparison, panel (a) shows the spatial distribution of $S$ at the same time when $\tau_{2}=0$. Obviously, it can be found that, without the influence of time delay, the density at each point of the system in space tends to be homogeneous, and with time delay, the system appears the spot pattern with irregular cold and hot spots coexisting, and the density at different hot spots and cold spots is also very uneven.

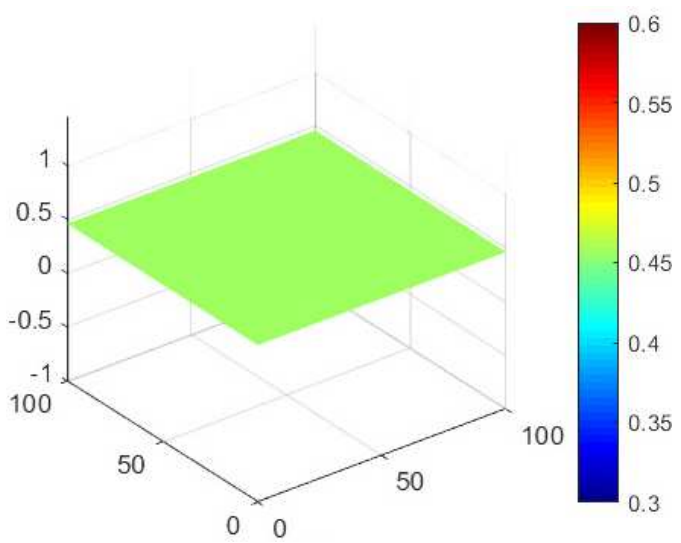

(a)

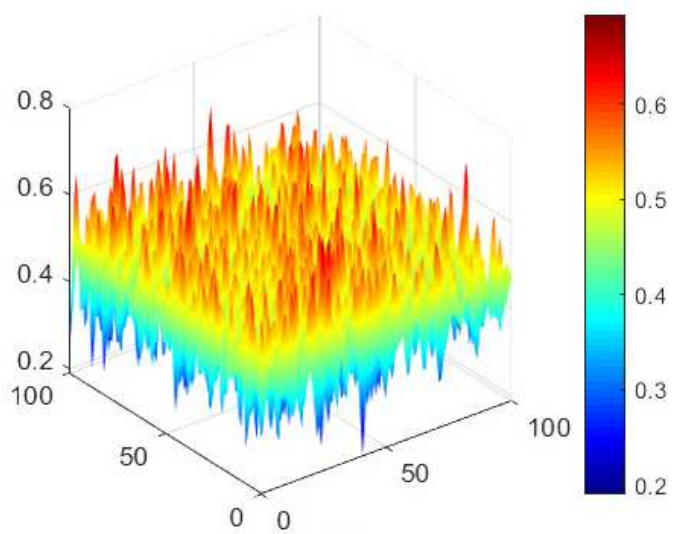

(b)

FIG. 6. Contrast of delay-induced pattern. In panel (a), $\tau_{2}=0, t=1000$. In panel (b), $\tau_{2}=0.25, t=1000$. The other parameters are set as follows: $K=1, A=0.1, \beta=0.46, \mu=0.5, d_{11}=0.5, d_{22}=0.5, d_{12}=24, d_{21}=0$.

\section{Pattern driven by period}

Firstly, in this part, By comparing Fig.7 with Fig.5, we can compare the effect of a small period on the pattern type of the original system. It can be found that, under the influence of periodic term, the pattern type and the time to stabilize did not change significantly. However, it is worth noting that in Fig.7(c) and Fig.5(c), the highly ordered distribution of hot spots is 
destroyed, and hot spots at the junction of irregular arrangements cannot reach the highest density of other hot spots even after a long time of evolution. Further, we present the pattern phenomenon driven by period. As shown in Fig.8(d), under this set of parameters, the necessary conditions for Turing instability can be met for any $\omega$ in the demonstrated range. $\omega$ is taken as 5 , Fig.8(a)-Fig.8(c) reflect the result of density evolution over time. At $t=600$, the strip appears. After the stable time, the spot pattern presents regular red and blue stripes, that is, the high-density and low-density strip areas are equidistant. Fig.8(f) shows a stereoscopic image of Fig.8(c), which is shaped like a colored cake! When $d_{11}^{\prime}=d_{22}^{\prime}=d_{12}^{\prime}=d_{21}^{\prime}=0$ and other parameters are the same, the system eventually tends to be stable over time, and the density of the stable time is shown in Fig.8(e).

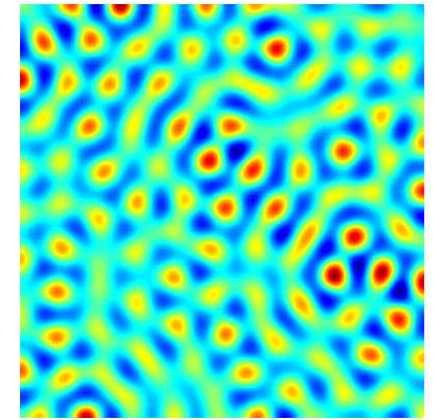

(a)
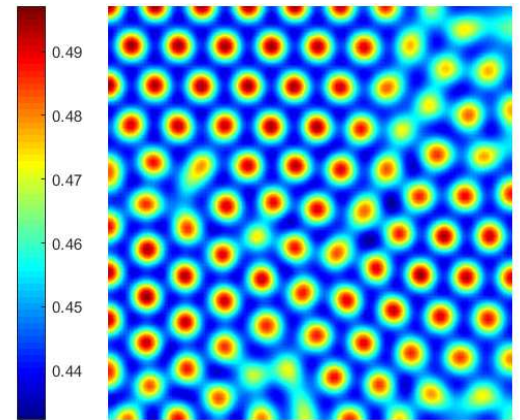

(b)
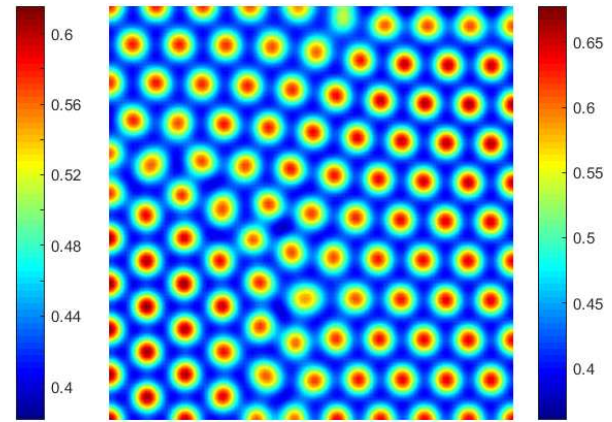

(c)

FIG. 7. The evolution process of Turing pattern for system (14) is shown. In panel (a), $t=2000$. In panel (b), $t=4000$. In panel (c), $t=6000$. The other parameters are set as follows: $K=1, A=0.1, \beta=0.46, \mu=0.5, d_{11}^{\prime}=0.07, d_{22}^{\prime}=0.07, d_{12}^{\prime}=0.014, d_{21}^{\prime}=0.135, d_{11}=0.5$, $d_{22}=0.5, d_{12}=0.1, d_{21}=0.965, \omega=0.5, \phi_{11}=0, \phi_{22}=\frac{3 \pi}{5}, \phi_{12}=\frac{\pi}{3}, \phi_{21}=\frac{\pi}{3}$. The parameter values of the overlap part are consistent with those in Fig.1 and Fig.5.

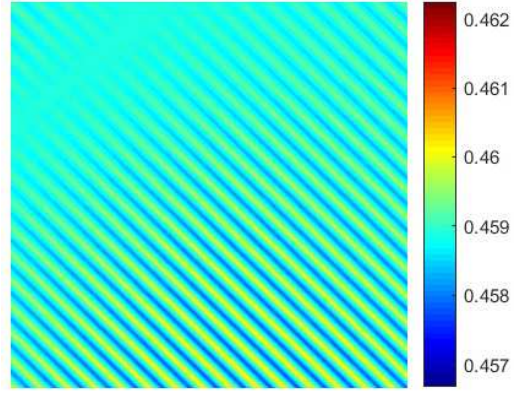

(a)

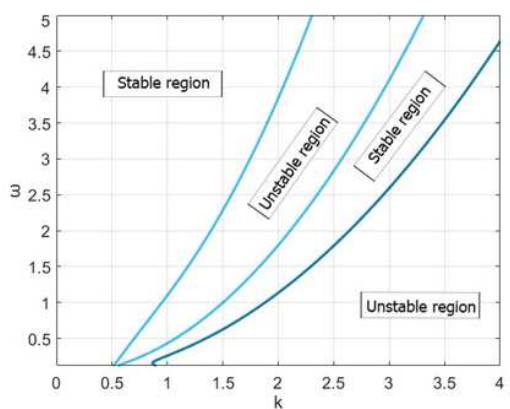

(d)

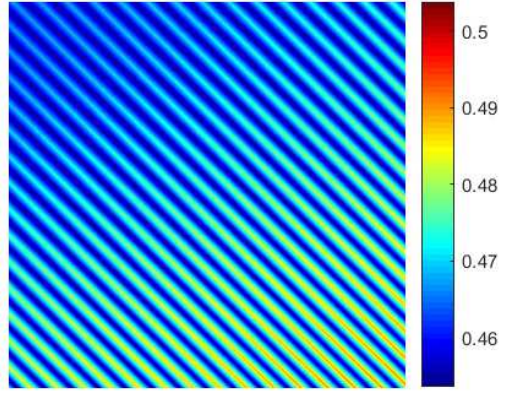

(b)

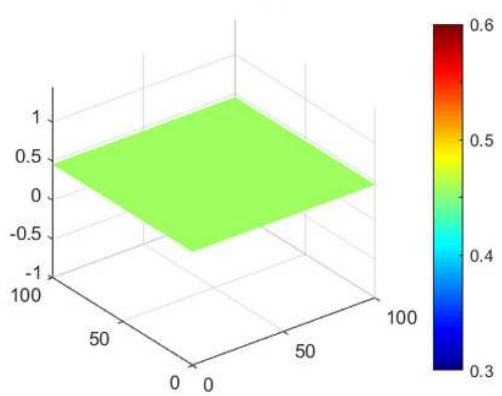

(e)

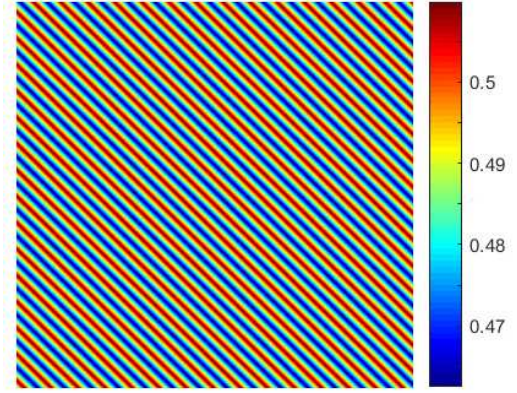

(c)

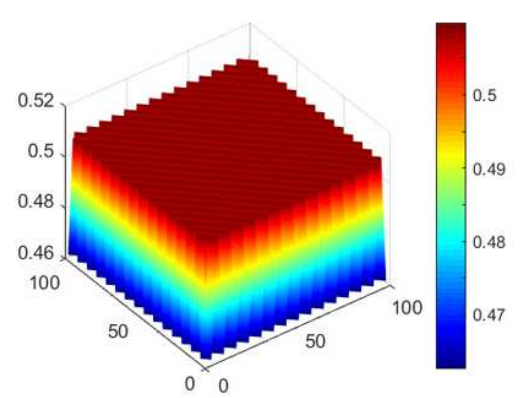

(f)

FIG. 8. Periodium-induced pattern versus periodium-free condition. Panel (d) shows the numerical curves of system (15) with periodic solutions changing with $\omega$ and $k$ when $K=1, A=0.1, \beta=0.46, \mu=0.5, d_{11}^{\prime}=1, d_{22}^{\prime}=1, d_{12}^{\prime}=0.2, d_{21}^{\prime}=1, d_{11}=0.5, d_{22}=0.5$, $d_{12}=0.1, d_{21}=0.5, \phi_{11}=0, \phi_{22}=\frac{4 \pi}{3}, \phi_{12}=\frac{\pi}{6}, \phi_{21}=\frac{\pi}{6}, \theta_{1}=0, \theta_{2}=0$. In the case of $\omega=5$, the formation of the pattern of system (14) over time is shown in panel (a) $(t=600)$, in panel (b) $(t=1200)$ and in panel (c) $(t=1800)$. Other parameters are the same as panel (d). Panel (f) gives the stereo image of panel (c). Meanwhile, panel (e) shows the stereo density distribution of $S$ at $t=1800$ without periodic influence $\left(d_{11}^{\prime}=d_{22}^{\prime}=d_{12}^{\prime}=d_{21}^{\prime}=0\right)$, and other parameters are the same as panel (d). 


\section{CONCLUSION}

Based on the characteristics of rumor propagation in cyberspace, this paper establishes a reaction-diffusion model for $S$ and $I$, studies and analyzes the diffusion behavior of various groups in cyberspace, and presents the characteristics of spatial instability under some special parameters. In the third section, Theorem 1 is used to solve the problem of local stability at the equilibrium point of a system with a small time delay. In the analysis of the influence of time delay on the system, we find that the smaller time delay generally does not change the positivity of the determinant of the jacobian matrix and the $H$ matrix of the original system, but it can affect the positivity of the $\operatorname{tr}(H)$, thus causing instability that does not exist in the original system in space. In the reaction-diffusion system with periodic coefficients, we can empoly Floquet's theory to analyze the stability of a firstorder approximate linear system with $k$ as the parameter, and determine whether the system satisfies the necessary condition of Turing instability by solving the parametric numerical solution satisfying the existence of periodic solution. In the numerical simulations, we have compared the types of patterns with and without cross diffusion in cause of $d_{22}>d_{11}$, and found that when $d_{12}=d_{21}=0$, the cold spot pattern is finally stable; when $d_{12}=0.2, d_{21}=0.4$, the cold spot-strip pattern is finally stable. When $d_{11}=d_{22}=0.5$, the hot spot pattern is finally stable. Furthermore, we give the delay-induced and period-induced patterns respectively, which proves the correctness that time delay and periodicity will lead to spatial instability that does not exist in the original system in the previous analysis.

These conclusions are helpful for us to better explore the spatial distribution status of all kinds of people in the network environment and provide help for the control and management of the network information environment. At the same time, the above research can also help to better understand the causes of spatial pattern formation and enrich the research paradigm of reaction-diffusion systems. In the future, we will continue to discuss the dynamics and control methods of spatiotemporal network rumor propagation ${ }^{42-46}$.

\section{ACKNOWLEDGEMENT}

This research is supported by National Natural Science Foundation of China (Grant No.12002135), Natural Science Foundation of Jiangsu Province, China (Grant No.BK20190836), China Postdoctoral Science Foundation (Grant No.2019M661732), and Natural Science Research of Jiangsu Higher Education Institutions of China (Grant No.19KJB110001).

\section{CONFLICT OF INTEREST}

The authors declare that they have no conflict of interest.

\section{REFERENCES}

${ }^{1}$ D. Varshney, D. K. Vishwakarma. A review on rumour prediction and veracity assessment in online social network. Expert Systems with Applications, 2020, 114208 .

${ }^{2}$ D. Daley, D. Kendall. Stochastic rumors. IMA Journal of Applied Mathematics, 1965, 1: 42-55.

${ }^{3}$ D. Maki, M. Thompson, Mathematical models and applications, with emphasis on the social. life and management sciences, 1973 , No. QA 402.M34

${ }^{4}$ L.A. Huo, L. Wang, X.M. Zhao. Stability analysis and optimal control of a rumor spreading model with media report. Physica A, $2019,517: 551-562$.

${ }^{5}$ J. Singha. A new analysis for fractional rumor spreading dynamical model in a social network with Mittag-Leffler law. Chaos, $2019,29: 013137$.

${ }^{6}$ L.H. Zhu, M.X. Liu, Y.M. Li. The dynamics analysis of a rumor propagation model in online social networks. Physica A, $2019,520: 118-137$.

${ }^{7}$ C.X. Wang, G.H. Wang, X.P. Luo, H. Li. Modeling rumor propagation and mitigation across multiple social networks. Physica A, 2019 , 535: 122240.

${ }^{8}$ Y.P. Xiao, L. Zhang, Q. Li, L. Liu. MM-SIS: Model for multiple information spreading in multiplex network. Physica A, $2019,513: 135-146$.

${ }^{9}$ L.H. Zhu, H.Y. Zhao, H.Y. Wang. Partial differential equation modeling of rumor propagation in complex networks with higher order of organization. Chaos, 2019, 29: 053106.

${ }^{10}$ A.Z. Yang, X.Y. Huang, X.M. Cai. X.F. Zhu, L. Lu. ILSR rumor spreading model with degree in complex network. Physica A, 2019,531 : 121807.

${ }^{11}$ J.R. Li, H.J. Jiang, X.H. Mei, C. Hu, G.L. Zhang. Dynamical analysis of rumor spreading model in multi-lingual environment and heterogeneous complex networks. Information Sciences, 2020, 536: 391-408.

${ }^{12}$ L.A. Huo, L. Wang, N.X. Song, C.Y. Ma, B. He. Rumor spreading model considering the activity of spreaders in the homogeneous network. Physica A, 2017, 468: 855-865.

${ }^{13}$ Y.Y. Cheng, L.A. Huo, L.J. Zhao. Rumor spreading in complex networks under stochastic node activity. Physica A, $2020,559: 125061$.

${ }^{14}$ Z.S. Wang, Q.T. Guo, S.W. Sun, C.Y. Xia. The impact of awareness diffusion on SIR-like epidemics in multiplex networks. Applied Mathematics and Computation, 2019, 349: 134-147.

${ }^{15}$ C. Granell, S. Gomez, A. Arenas. Dynamical interplay between awareness and epidemic spreading in multiplex networks. Physical Review Letters, 2013, 111: 128701.

${ }^{16}$ C.Y. Xia, Z.S. Wang, C.Y. Zheng, Q.T. Guo, Y.T. Shi. M. Dehmer, Z.Q. Chen. A new coupled disease-awareness spreading model with mass media on multiplex networks. Information Sciences, 2019, 471: 185-200.

${ }^{17}$ Turing AM. On the chemical basis of morphogenesis. Philos Trans Roy Soc London B, 1952, 237: $37-72$. 
${ }^{18}$ Shigesada N, Kawasaki K, Teramoto E. Spatial segregation of interacting species. Journal of Theoretical Biology, 1979, 1: 83-99.

${ }^{19}$ B. Hasslacher, R. Kapral, A. Lawniczak. Molecular Turing structures in the biochemistry of the cell. Chaos, 1993, 3: 7.

${ }^{20}$ J. M. Chung, E. Peacock-Lopeza. Bifurcation diagrams and Turing patterns in a chemical self-replicating reaction-diffusion system with cross diffusion. J. Chem. Phys., 2007, 127: 174903.

${ }^{21}$ Q.S. Li, L. Ji. Control of Turing pattern by weak spatial perturbation. Journal of Chemical Physics, 2004, 120: 9690-9693.

${ }^{22}$ Z.G. Mao, Z.Q. Yin, J.X. Ran. Generation of high-power-density atmospheric pressure plasma with liquid electrodes. Applied Physics Letters, $2004,84: 5142$.

${ }^{23}$ B. Liu, R.C. Wu, L.P Chen. Turing-Hopf bifurcation analysis in a superdiffusive predator-prey model. Chaos, 2018, $28: 113118$.

${ }^{24}$ B.X. Zhang, Y.L. Cai, B.X. Wang, W.M. Wang. Pattern formation in a reaction-diffusion parasite-host model. Physica A, 2019, 525: 732-740.

${ }^{25}$ S.T. Ghorai, S. Poria. Turing patterns induced by cross-diffusion in a predator-prey system in presence of habitat complexity. Chaos, Solitons \& Fractals, 2016, 91: 421-429.

${ }^{26} \mathrm{C}$. Tiana. Delay-driven spatial patterns in a plankton allelopathic system. Chaos, 2012, 22: 013129.

${ }^{27}$ H.J. Wang, Z. Ren. Competition of spatial and temporal instabilities under time delay near codimension-two Turing-Hopf bifurcations. Communications in Theoretical Physics, 2011, 56: 339.

${ }^{28}$ X.Z. Lian, H.L. Wang, W.M. Wang. Delay-driven pattern formation in a reaction-diffusion predator-prey model incorporating a prey refuge. Journal of Statal Mechanics: Theory and Experiment, 2013, P04006.

${ }^{29}$ Byliole S. Djouda, F. M. Moukam Kakmeni, P. Guemkam Ghomsi. Theoretical analysis of spatial nonhomogeneous patterns of entomopathogenic fungi growth on insect pest. Chaos, 2019, 29: 053134.

${ }^{30}$ D. W. Jordan, P. Smith, Nonlinear Ordinary Differential Equations. Oxford University Press Inc., New York, 2007.

${ }^{31}$ R. Yamapi, P. Woafo. Synchronized states in a ring of four mutually coupled self-sustained electromechanical devices. Communications in Nonlinear Science and Numerical Simulation, 2006, 11: 186-202.

${ }^{32}$ R. Yamapi, P. Woafo. Dynamics and synchronization of coupled self-sustained electromechanical devices. Journal of Sound and Vibration, 2005, 285: 11511170 .

${ }^{33}$ Y. Chembo Kouomou, P. Woafo. Stability and chaos control in electrostatic transducers. Physica Scripta, 2000, 62: 255.

${ }^{34}$ L.L. Chang, C. Liu, G.Q. Sun, Z. Wang, Z. Jin. Delay-induced patterns in a predator-prey model on complex networks with diffusion. New Journal of Physics, 2019, 21: 073035.

${ }^{35}$ M. Zheng, B. Shao, Q. Ouyang. Identifying network topologies that can generate turing pattern. Journal of Theoretical Biology, 2016, $408: 88-96$.

${ }^{36}$ Y. Liu, S.G. Ruan, L. Yang. Stability transition of persistence and extinction in an avian influenza model with Allee effect and stochasticity. Communications in Nonlinear Science and Numerical Simulation, 2020, 91: 105416

${ }^{37}$ A.M. Kramer, B. Dennis, A.M. Liebhold, J.M. Drake. The evidence for Allee effects. Population Ecology, 2009, 51: 341-354.

${ }^{38}$ M. Krstic, M. Jovanovic. On stochastic population model with the Allee effect. Mathematical and Computer Modelling, 2010, 52: 370-379.

${ }^{39}$ X.Y. Gao, L.X. Tian, W.Y. Li. Coupling interaction impairs knowledge and green behavior diffusion in complex networks. Journal of Cleaner Production, 2020, 249: 119419.

${ }^{40}$ L.H. Zhu, M.T. Zhou, Z.D. Zhang. Dynamical analysis and control strategies of rumor spreading models in both homogeneous and heterogeneous networks. Journal of Nonlinear Science, 2020, 30:2545-2576.

${ }^{41}$ J. Li, H.L. Wang, Q. Ouyang. Square Turing patterns in reaction-diffusion systems with coupled layers. Chaos, 2014, $24: 023115$.

${ }^{42}$ L.H. Zhu, G. Guan, Y.M. Li. Nonlinear dynamical analysis and control strategies of a network-based SIS epidemic model with time delay. Applied Mathematical Modelling, 2019, 70: 512-531.

${ }^{43}$ J. Hua, L.X. An, Y.M. Li. Bionic fuzzy sliding mode control and robustness analysis. Applied Mathematical Modelling, 2015, $39: 4482-4493$.

${ }^{44}$ F.X. Zhang, J. Hua, Y.M. Li. Indirect adaptive fuzzy control of SISO nonlinear systems with input-output nonlinear relationship. IEEE Transactions on Fuzzy Systems, 2018, 26: 2699-2708.

${ }^{45}$ J.W. Huo, Y.M. Li. J. Hua. Global dynamics of SIRS model with no full immunity on semidirected networks. Mathematical Problems in Engineering, 2019, 8792497.

${ }^{46}$ P. Miao, Z.D. Zhang, C.W. Lim, X.D. Wang. Hopf bifurcation and hybrid control of a delayed ecoepidemiological model with nonlinear incidence rate and Holling type II functional response. Mathematical Problems in Engineering, 2018, 2018: 6052503. 
Figures

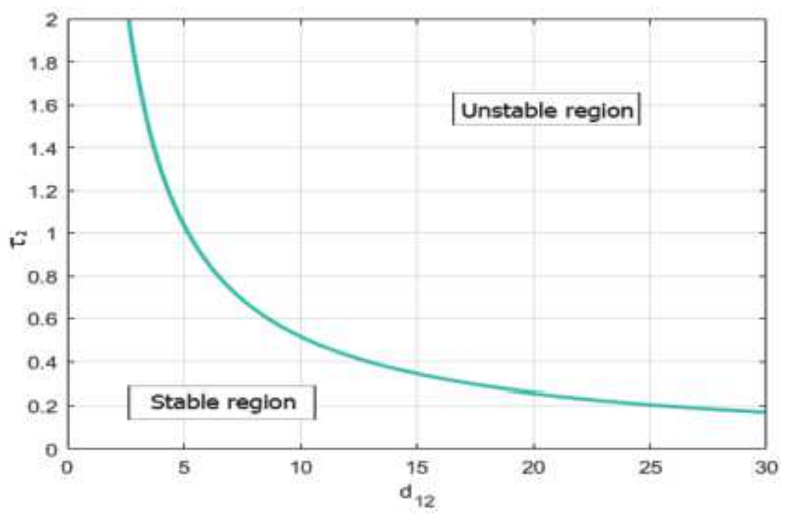

FIG. 1. Threshold curve of Turing instability induced by $\tau_{2}$ changing with $d_{12}$ in the approximation system of system (9). Other parameters are set as follows: $K=1, A=0.1, \beta=0.46, \mu=0.5, d_{11}=0.5, d_{22}=0.5, d_{21}=0$.

\section{Figure 1}

Please view the figure caption in the figure. 


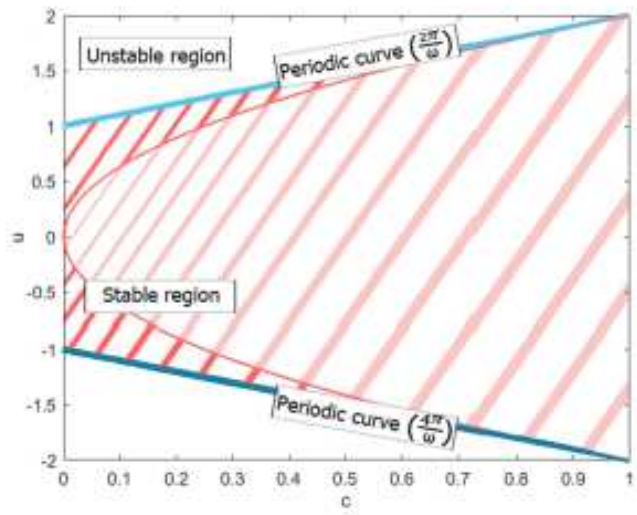

(a)

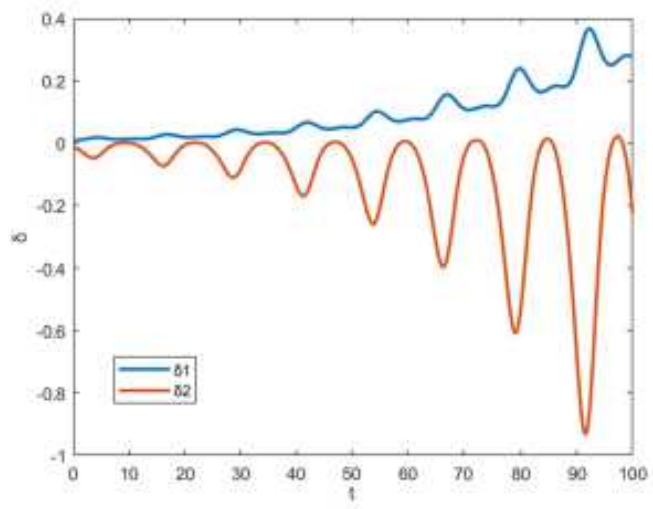

(c)

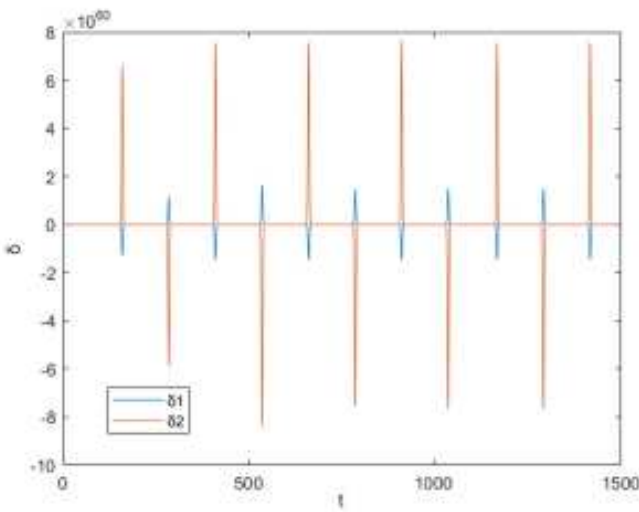

(e)

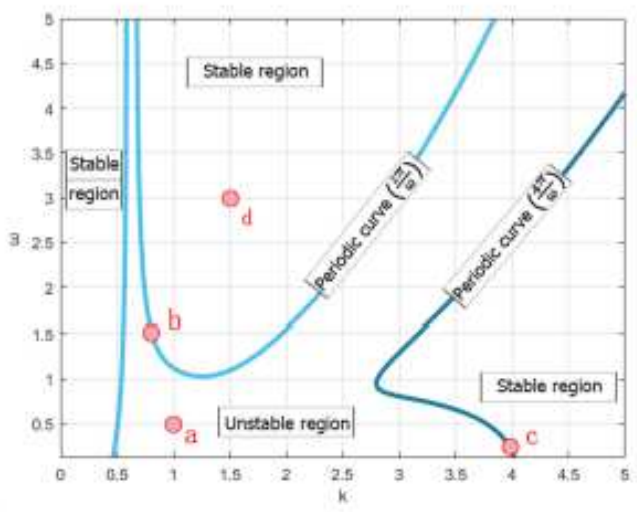

(b)

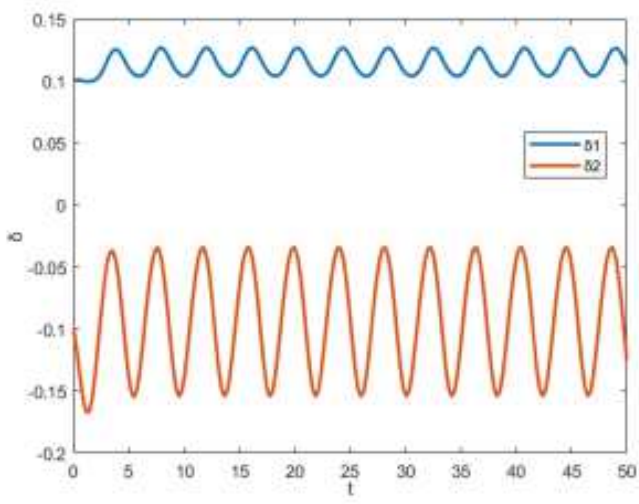

(d)

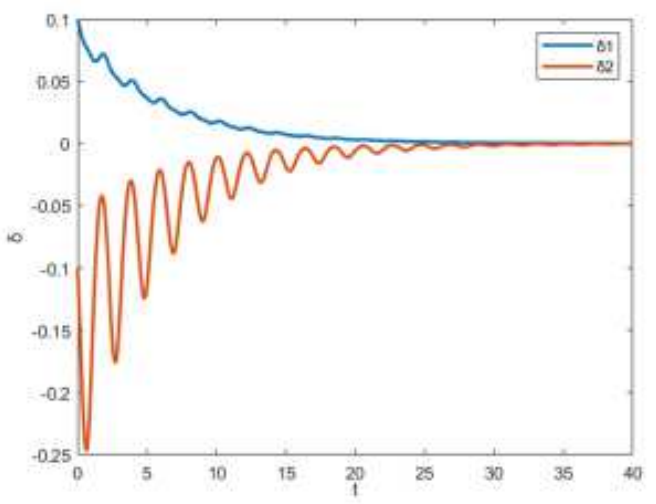

(f)

FIG. 2. Panel (b) shows the state of system (15) under different values of $u$ and $c$. Panel (b) shows the state of system (15) under different values of $k$ and $\omega$. Panel (c)-(f) show some special solution curves whose parameters correspond to the four points $a(k=1, \omega=0.5), b$ $(k=0.8, \omega=1.53), c(k=4, \omega=0.05), d(k=1.5, \omega=3)$ marked in panel (b) respectively. In panel (b)-(f), the other parameters are set as follows: $K=1, A=0.1, \beta=0.46, \mu=0.5, d_{11}=d_{11}^{\prime}=0.5, d_{22}=d_{22}^{\prime}=0.5, d_{12}=d_{12}^{\prime}=0.1, d_{21}=d_{21}^{\prime}=0.965, \phi_{11}=0, \phi_{22}=\frac{4 \pi}{3}$, $\phi_{12}=\frac{\pi}{6}, \phi_{21}=\frac{\pi}{6}, \theta_{1}=0, \theta_{2}=0$.

\section{Figure 2}

Please view the figure caption in the figure. 


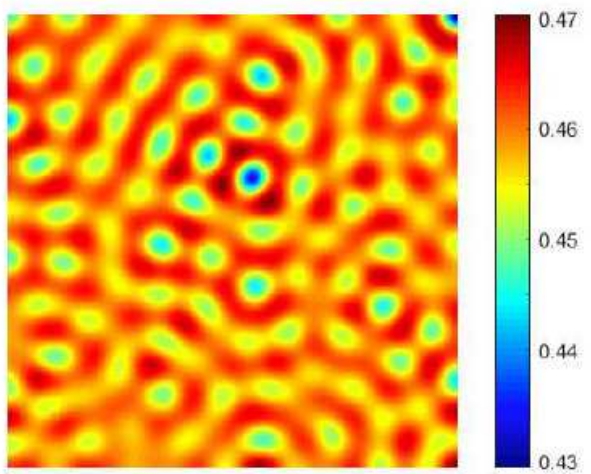

(a)

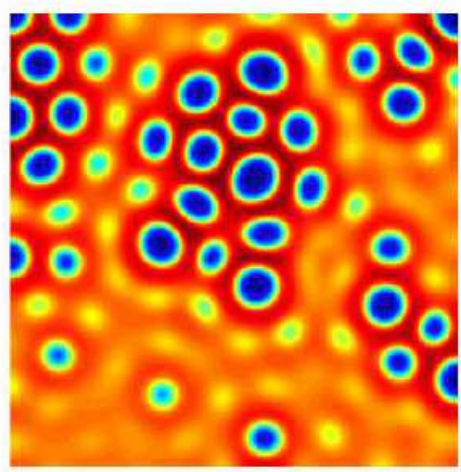

(b)

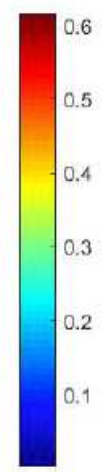

FIG. 3 . In the case that $d_{11}=0.1, d_{22}=2, d_{12}=d_{21}=0$, the evolution process of Turing pattern for system (3) is shown. In panel (a), $t=250$. In panel (b), $t=500$. In panel (c), $t=750$. The other parameters are set as follows: $K=1, A=0.1, \beta=0.46, \mu=0.5$.

\section{Figure 3}

Please view the figure caption in the figure.

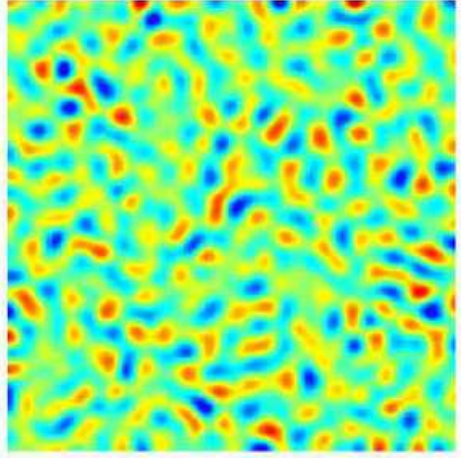

(a)

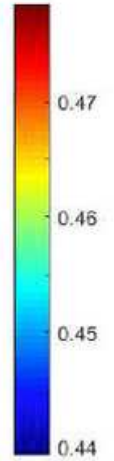

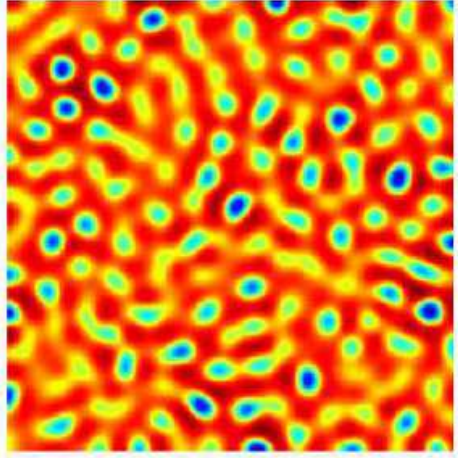

(b)
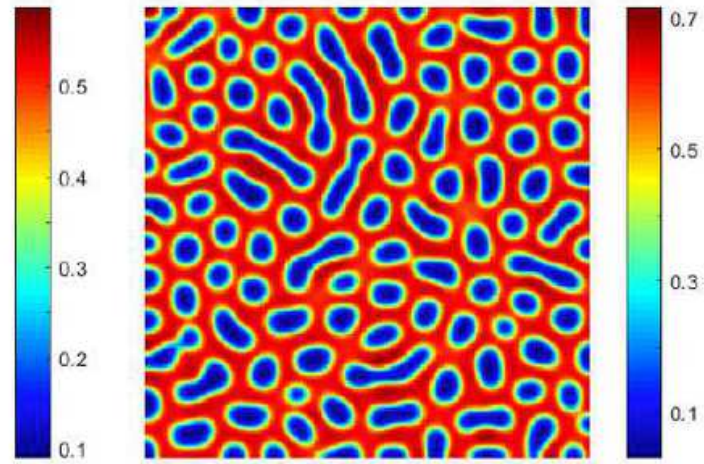

(c)

FIG. 4. In the case that $d_{11}=0.1, d_{22}=2, d_{12}=0.2, d_{21}=0.4$, the evolution process of Turing pattern for system (3) is shown. In panel (a), $t=20$. In panel (b), $t=40$. In panel (c), $t=60$. The other parameters are set as follows: $K=1, A=0.1, \beta=0.46, \mu=0.5$.

\section{Figure 4}

Please view the figure caption in the figure. 


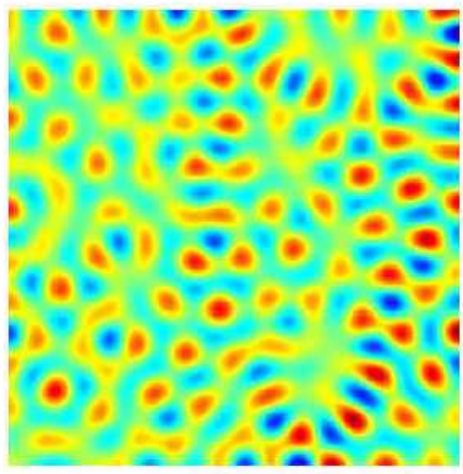

(a)
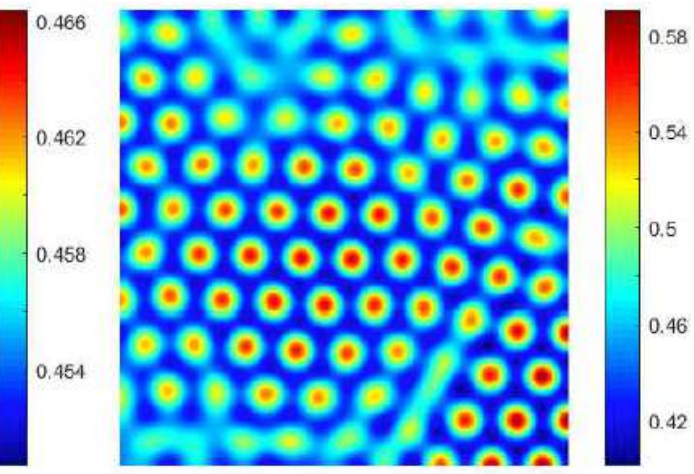

(b)

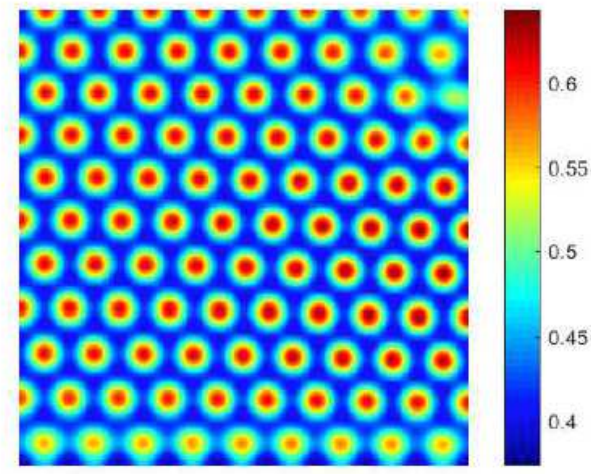

(c)

FIG. 5. In the case that $d_{11}=0.5, d_{22}=0.5, d_{12}=0.1, d_{21}=0.965$, the evolution process of Turing pattern for system (2) is shown. In panel (a), $t=2000$. In panel (b), $t=4000$. In panel (c), $t=6000$. The other parameters are set as follows: $K=1, A=0.1, \beta=0.46, \mu=0.5$.

\section{Figure 5}

Please view the figure caption in the figure.

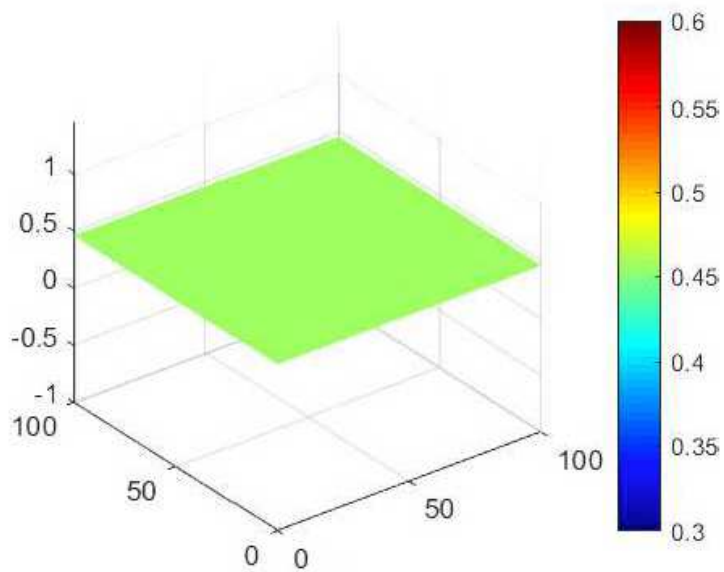

(a)

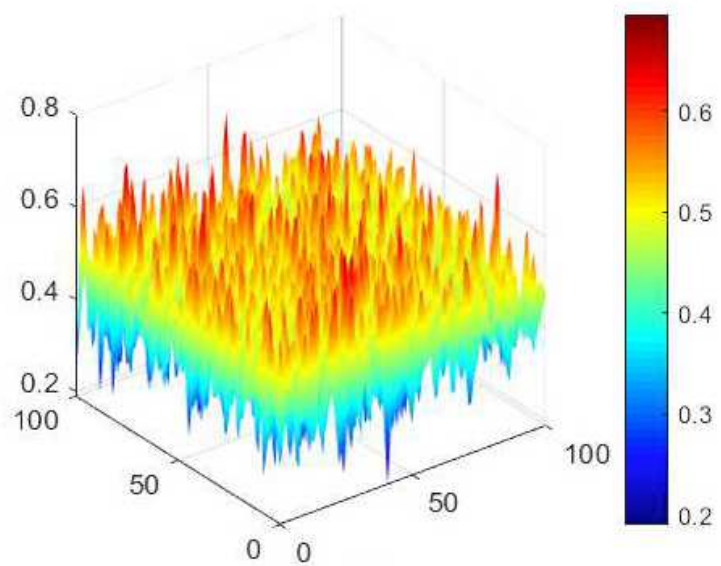

(b)

FIG. 6. Contrast of delay-induced pattern. In panel (a), $\tau_{2}=0, t=1000$. In panel (b), $\tau_{2}=0.25, t=1000$. The other parameters are set as follows: $K=1, A=0.1, \beta=0.46, \mu=0.5, d_{11}=0.5, d_{22}=0.5, d_{12}=24, d_{21}=0$.

\section{Figure 6}

Please view the figure caption in the figure. 


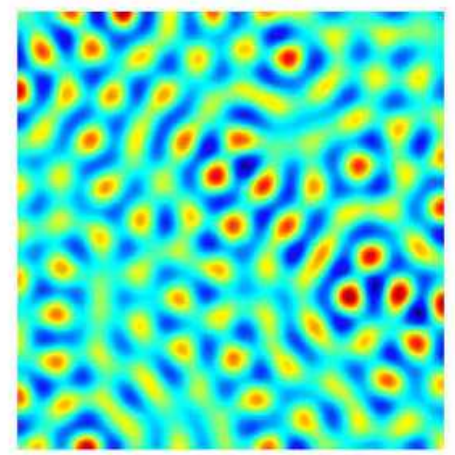

(a)

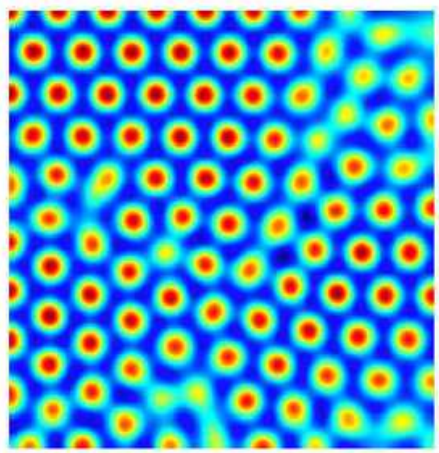

(b)

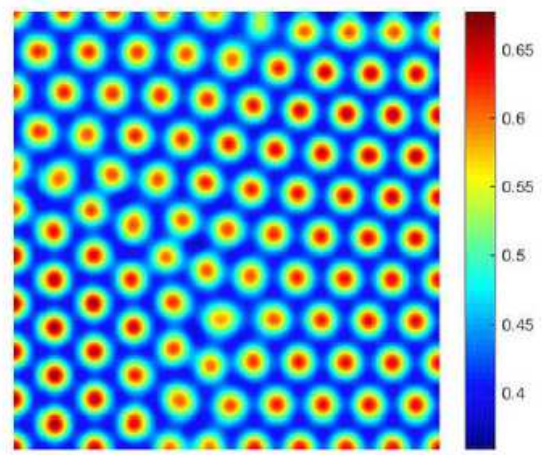

(c)

FIG. 7. The evolution process of Turing pattern for system (14) is shown. In panel (a), $t=2000$. In panel (b), $t=4000$. In panel (c), $t=6000$. The other parameters are set as follows: $K=1, A=0.1, \beta=0.46, \mu=0.5, d_{11}^{\prime}=0.07, d_{22}^{\prime}=0.07, d_{12}^{\prime}=0.014, d_{21}^{\prime}=0.135, d_{11}=0.5$, $d_{22}=0.5, d_{12}=0.1, d_{21}=0.965, \omega=0.5, \phi_{11}=0, \phi_{22}=\frac{3 \pi}{5}, \phi_{12}=\frac{\pi}{3}, \phi_{21}=\frac{\pi}{3}$. The parameter values of the overlap part are consistent with those in Fig.1 and Fig.5.

\section{Figure 7}

Please view the figure caption in the figure.

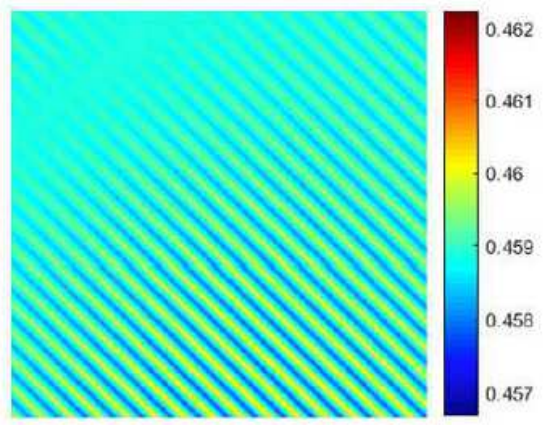

(a)

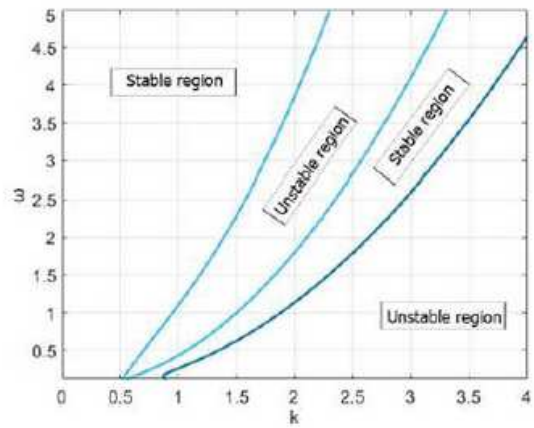

(d)

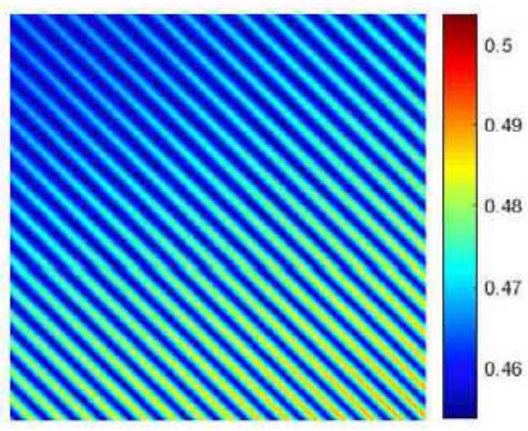

(b)

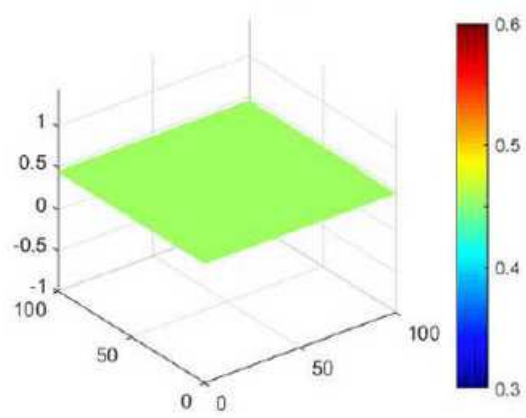

(e)

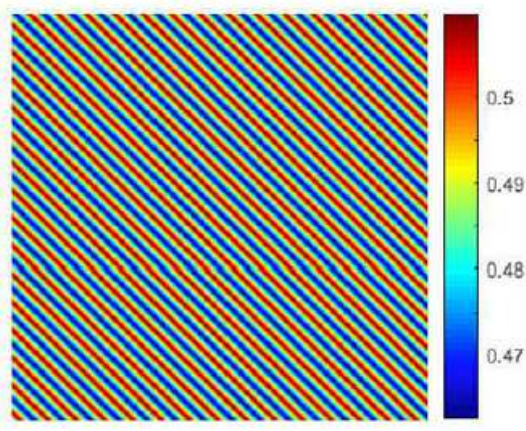

(c)

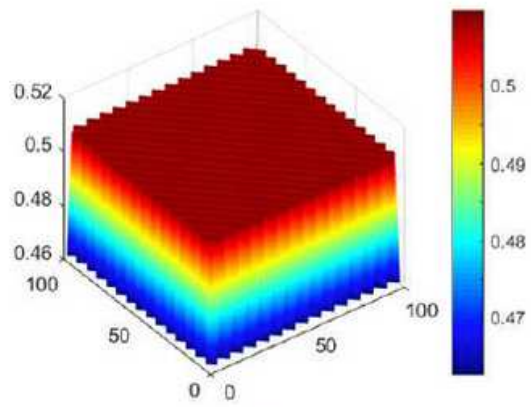

(f)

FIG. 8. Periodium-induced pattern versus periodium-free condition. Panel (d) shows the numerical curves of system (15) with periodic solutions changing with $\omega$ and $k$ when $K=1, A=0.1, \beta=0.46, \mu=0.5, d_{11}^{\prime}=1, d_{22}^{\prime}=1, d_{12}^{\prime}=0.2, d_{21}^{\prime}=1, d_{11}=0.5, d_{22}=0.5$, $d_{12}=0.1, d_{21}=0.5, \phi_{11}=0, \phi_{22}=\frac{4 \pi}{3}, \phi_{12}=\frac{\pi}{6}, \phi_{21}=\frac{\pi}{6}, \theta_{1}=0, \theta_{2}=0$. In the case of $\omega=5$, the formation of the pattern of system (14) over time is shown in panel (a) $(t=600)$, in panel (b) $(t=1200)$ and in panel (c) $(t=1800)$. Other parameters are the same as panel (d). Panel (f) gives the stereo image of panel (c). Meanwhile, panel (e) shows the stereo density distribution of $S$ at $t=1800$ without periodic influence $\left(d_{11}^{\prime}=d_{22}^{\prime}=d_{12}^{\prime}=d_{21}^{\prime}=0\right)$, and other parameters are the same as panel (d).

\section{Figure 8}

Please view the figure caption in the figure. 